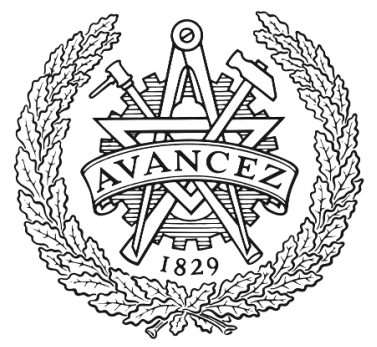

CHALMERS

UNIVERSITY OF TECHNOLOGY

\title{
Role of large-scale advection and small-scale turbulence on vertical migration of gyrotactic swimmers
}

Downloaded from: https://research.chalmers.se, 2023-04-26 14:28 UTC

Citation for the original published paper (version of record):

Marchioli, C., Bhatia, H., Sardina, G. et al (2019). Role of large-scale advection and small-scale turbulence on vertical migration of gyrotactic swimmers. Physical Review Fluids, 4. http://dx.doi.org/10.1103/PhysRevFluids.4.124304

N.B. When citing this work, cite the original published paper. 


\title{
Role of large-scale advection and small-scale turbulence on vertical migration of gyrotactic swimmers
}

\author{
C. Marchioli, ${ }^{1,2,{ }^{*}}$ H. Bhatia $\odot,{ }^{1}$ G. Sardina,${ }^{3}$ L. Brandt, ${ }^{4}$ and A. Soldati ${ }^{1,5}$ \\ ${ }^{1}$ Department of Engineering and Architecture, University of Udine, 33100 Udine, Italy \\ ${ }^{2}$ Department of Fluid Mechanics, CISM, 33100 Udine, Italy \\ ${ }^{3}$ Division of Fluid Dynamics, Department of Mechanics and Maritime Sciences, \\ Chalmers University of Technology, 41258 Gothenburg, Sweden \\ ${ }^{4}$ Linnè FLOW Centre and SeRC, KTH Mechanics, SE 10044, Stockholm, Sweden \\ ${ }^{5}$ Institute of Fluid Mechanics and Heat Transfer, TU Wien, 1040 Wien, Austria
}

(Received 25 June 2019; published 9 December 2019)

\begin{abstract}
In this work, we use direct-numerical-simulation-based Eulerian-Lagrangian simulations to investigate the dynamics of small gyrotactic swimmers in free-surface turbulence. We consider open-channel flow turbulence in which bottom-heavy swimmers are dispersed. Swimmers are characterized by different vertical stability, so that some realign to swim upward with a characteristic time smaller than the Kolmogorov timescale, while others possess a reorientation time longer than the Kolmogorov timescale. We cover one order of magnitude in the flow Reynolds number and two orders of magnitude in the stability number, which is a measure of bottom heaviness. We observe that large-scale advection dominates vertical motion when the stability number, scaled on the local Kolmogorov timescale of the flow, is larger than unity: This condition is associated to enhanced migration toward the surface, particularly at low Reynolds number, when swimmers can rise through surface renewal motions that originate directly from the bottomboundary turbulent bursts. Conversely, small-scale effects become more important when the Kolmogorov-based stability number is below unity: Under this condition, migration toward the surface is hindered, particularly at high Reynolds, when bottom-boundary bursts are less effective in bringing bulk fluid to the surface. In an effort to provide scaling arguments to improve predictions of models for motile microorganisms in turbulent water bodies, we demonstrate that a Kolmogorov-based stability number around unity represents a threshold beyond which swimmer capability to reach the free surface and form clusters saturates.
\end{abstract}

DOI: 10.1103/PhysRevFluids.4.124304

\section{INTRODUCTION}

Motile microorganisms have developed different ways of adapting to aquatic flows. Some organisms, like raphidophytes and dinoflagellates phytoplankton species, are able to migrate upward toward well-lit surface waters during the day (to activate photosynthesis, absorb $\mathrm{CO}_{2}$, and produce oxygen), and downward toward nutrient-rich deeper layers at night [1]. This ability is deemed to be an important cause of the succession between motile and nonmotile species when conditions are turbulent [2]. It is now well-known that vertical migration is favored by the capability of swimmers to change the direction of propulsion in response to different physicochemical biases, e.g., chemotaxis, gyrotaxis, and phototaxis (see Refs. [3-6] and references therein for a

\footnotetext{
*Corresponding author: marchioli@uniud.it
} 
detailed discussion of the interactions that swimmers have with such stimuli and on the resulting bioconvection processes). Since responses to such external stimuli act across a wide spectrum of timescales, the interplay between biased motility and the timescale of the perturbations originating from the background carrier flow may lead to complex dynamics: When the flow is turbulent, in particular, nontrivial collective behavior (like aggregation into fractal clusters $[7,8]$ or thin shear layers $[9,10]$ and even morphological adaptations [1]) can be observed.

Among the most widely studied motile responses, one is gyrotaxis: Gyrotaxis originates from the competition between a stabilising gravitational torque, due to an asymmetric mass distribution of the swimmer that favors its orientation along the direction of gravity (gravitaxis), and a destabilizing viscous torque, produced by the local shear acting on the swimmer $[5,11,12]$. The role of gyrotaxis in determining the transport properties of self-propelled microorganisms has been investigated both in laminar [13-15] and in turbulent flows [7,16-22]. Turbulence, in particular, leads to preferential sampling of downwelling and upwelling regions in the bulk of the flow [7,21-23], small-scale fractal patchiness $[16,17,24]$, and shear-induced trapping that may hinder vertical migration $[18,20]$. All these processes are significantly influenced by the multiscale structure of the flow, which encompasses large-scale advective motions generated by coherent structures as well as small-scale motions associated with the local fluid velocity gradients. Recently, Borgnino et al. [22] have shown that, for homogeneous isotropic turbulence, the large (integral) flow scales control the transition from preferential sampling of downwelling regions to preferential sampling of upwelling regions at sufficiently high Reynolds number, whereas the small scales control fractal clustering. Besides these findings, however, we are not aware of further studies aiming to understand the relative importance of the flow scales on swimmer dynamics (and on vertical migration, in particular). As a consequence, a consistent cross-timescale framework connecting turbulence and swimming strategy is still lacking. In this paper, we provide a first contribution to the development of such framework by examining the surfacing of gyrotactic swimmers toward a free surface through a three-dimensional nonhomogeneous turbulent flow. Specifically, we consider swimmers with different gyrotactic bias and self-propelling speeds that are typical of the most common phytoplankton species, and we track their motion in different flow realizations (namely, different Reynolds numbers) in an open channel, which mimic a fluctuating heterogeneous environment characterized by a marked separation between the forcing scale and the dissipation scale.

A convenient way to parametrize the relative importance of the flow scales on the swimmer dynamics is provided by the stability number, $\Psi$, defined as the inverse of the dimensionless time a perturbed gravitactic swimmer takes to return to the vertical orientation in still fluid [11,25]: High (respectively, low) stability number represents the case of a swimmer with small (respectively, large) reorientation time, which can hardly (respectively, easily) be destabilized by the background flow. Indicating with $\tau_{K}$ the dimensionless Kolmogorov timescale of the flow, three different physical instances can be highlighted [18,20]: (i) $\Psi \tau_{K} \ll 1$, representing the case in which the timescale of gravitaxis is large compared to the dissipative flow timescale, and swimmers dynamics are primarily controlled by the external action of turbulence; (ii) $\Psi \tau_{K} \gg 1$, representing the case in which the timescale of gravitaxis is small compared to the timescale of the smallest turbulent eddies, and swimmers dynamics are controlled by their fast response to gravity; (iii) $\Psi \tau_{K} \sim \mathcal{O}$ (1), representing the situation in which the two timescales are comparable, and the motion of the swimmers results from the competition between turbulence and gravitational bias.

We perform a systematic investigation of these cases, and we cover two orders of magnitude for the stability number and one order of magnitude for the flow Reynolds number to highlight the role of large-scale advection and small-scale turbulence under significantly different flow conditions. More specifically, for the lower Reynolds number considered, the structure of the flow at the free surface is strongly influenced by near-wall turbulence activity [26-28]. In this low Reynolds number case, the thickness of the sublayer attached to the free surface is a significant fraction of the integral length scale, and that the surface-influenced region overlaps the logarithmic layer induced by the bottom shear, with the consequence that the turbulent field 'seen' by the surface is strongly anisotropic. Conversely, for the highest Reynolds number, the near-surface turbulence has nearly 


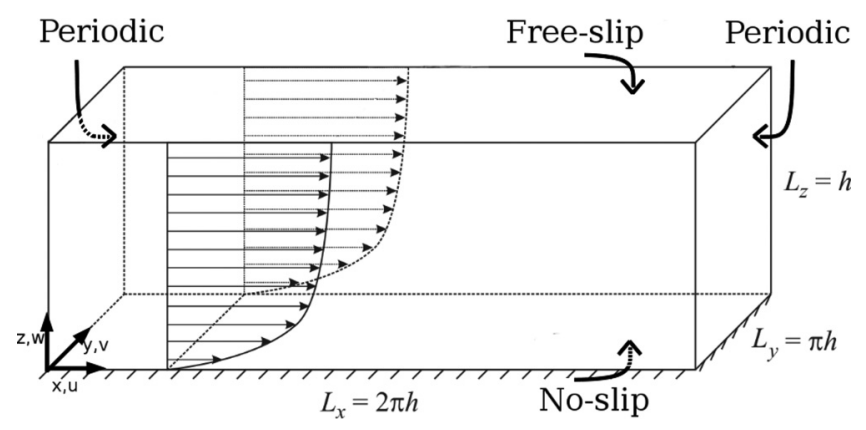

FIG. 1. Sketch of the computational domain with boundary conditions for the fluid.

lost the memory of the bottom boundary layer within which it was generated, and the anisotropy of the turbulence entering the surface region has decreased significantly [28]. Such difference in the flow phenomenology is expected to influence the pace at which swimmers rise toward the surface and their tendency to form highly-concentrated clusters.

The rest of the paper is organised as follows: The problem statement, the governing equations and the numerical methodology used for the simulations are presented in Sec. II; Sec. III is devoted to the analysis and discussion of large- and small-scale effects on swimmer surfacing and clustering at varying scale separation. Finally, concluding remarks are drawn in Sec. IV.

\section{PHYSICAL PROBLEM AND METHODOLOGY}

To study swimmer surfacing and clustering, we performed direct numerical simulations of turbulent open-channel flow with a nondeformable, flat free surface. The effect of imposing the flat surface does not alter the turbulence in the bulk of flow, as it is constantly generated from the bottom wall (source of shear). The choice of imposing a flat free surface has been discussed in Lovecchio et al. [18] and is motivated by previous findings [24,29-31], which have shown that light particles moving at the deformed free surface of a turbulent flow are subject to clustering mechanisms that come from the horizontal divergence at the surface: These mechanisms induce a compressible effect similar to the one observed over flat surfaces.

The reference geometry, shown in Fig. 1, consists of two horizontal (infinite) flat parallel boundaries, one being the bottom wall and the other the free surface, with the $x, y$, and $z$ axes of the coordinate system pointing in the streamwise, spanwise, and wall-normal directions, respectively. Indicating with $h$ the channel height, the size of the channel is $2 \pi h \times \pi h \times h$ in $x, y$, and $z$, respectively.

The flow field is calculated by integrating the three-dimensional incompressible continuity and Navier-Stokes equations. In dimensionless vector form, these equations read as

$$
\nabla \cdot \mathbf{u}=0
$$

$$
\frac{\partial \mathbf{u}}{\partial t}+\mathbf{u} \cdot \nabla \mathbf{u}=-\nabla p+\delta_{p}+\frac{1}{\operatorname{Re}_{\tau}} \nabla^{2} \mathbf{u}
$$

with $\mathbf{u}=\left(u_{x}, u_{y}, u_{z}\right)$ the fluid velocity, $p$ the fluctuating kinematic pressure, $\delta_{p}=(1,0,0)$ the mean pressure gradient that drives the flow in the streamwise direction, and $\operatorname{Re}_{\tau}=h u_{\tau} / v$ the shear Reynolds number based on the shear velocity $u_{\tau}=\sqrt{h\left|\delta_{p}\right| / \rho}$, and on the kinematic fluid viscosity, $v$. Equations (1) and (2) are solved directly using a pseudospectral method that transforms field variables into wave-number space, through Fourier representations for the streamwise and spanwise directions (using $k_{x}$ and $k_{y}$ wave numbers, respectively) and a Chebyshev representation for the wall-normal nonhomogeneous direction (using $T_{n}$ coefficients). A two-level explicit AdamsBashforth scheme for the nonlinear terms and an implicit Crank-Nicolson method for the viscous 


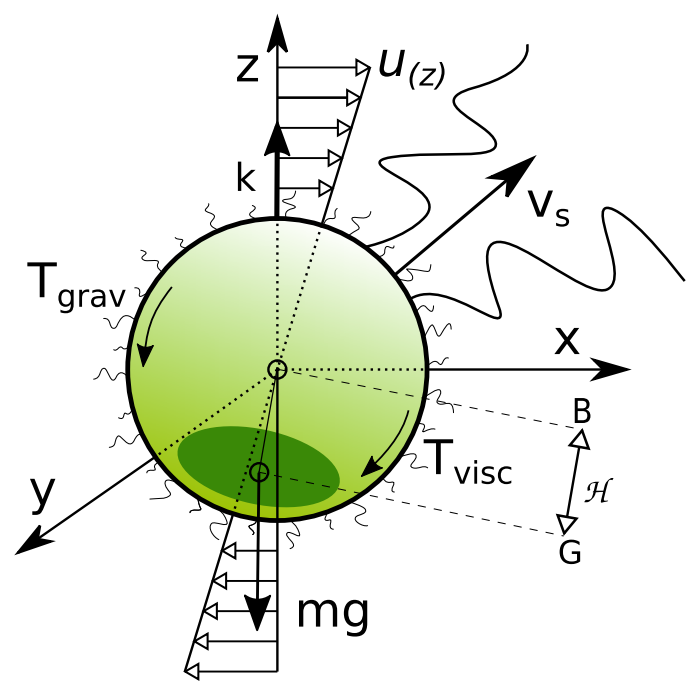

FIG. 2. Gyrotactic microorganisms swim with velocity $v_{s}$ in the direction given by the orientation vector $\mathbf{p}$, set by a balance of torques: The torque due to cell asymmetry (bottom heaviness: $\mathbf{T}_{\text {grav }}$ ), which tends to align the cell to its preferential orientation along the vertical direction $\mathbf{k}$, and the torque due to the flow $\mathbf{T}_{\text {visc }}$.

terms are employed for time advancement. The convective nonlinear terms are first computed in the physical space and then transformed in the wave-number space using a dealiasing procedure based on the 2/3-rule; derivatives are evaluated directly in the wave-number space to maintain spectral accuracy. Further details can be found in Lovecchio et al. [24]. For the fluid velocity, periodic boundary conditions are applied in $x$ and $y$, whereas no-slip boundary conditions are enforced at the bottom wall $\left(u_{x}=u_{y}=u_{z}=0\right)$. At the free surface, standard no-stress conditions $\left(\partial u_{x} / \partial z=0\right.$, $\partial u_{y} / \partial z=u_{z}=0, u_{z}=0$ ) are used. A campaign of direct numerical simulations was performed to investigate the motion of the swimmers at increasing turbulence intensity. Results presented in this paper are relative to three values of the shear Reynolds number: $\operatorname{Re}_{\tau}^{L}=170, \operatorname{Re}_{\tau}^{I}=510$, and $\mathrm{Re}_{\tau}^{H}=1020$ corresponding, respectively, to shear velocity $u_{\tau}^{L}=0.003 \mathrm{~m} \mathrm{~s}^{-1}, u_{\tau}^{I}=0.009 \mathrm{~m} \mathrm{~s}^{-1}$ and $u_{\tau}^{H}=0.018 \mathrm{~m} \mathrm{~s}^{-1}$ in a channel with $h=6 \mathrm{~cm}$. The size of the computational domain in wall units is $L_{x}^{+} \times L_{y}^{+} \times L_{z}^{+}=2 \pi \operatorname{Re}_{\tau} \times \pi \operatorname{Re}_{\tau} \times \operatorname{Re}_{\tau}$, discretized in physical space with $N_{x} \times N_{y} \times$ $N_{z}=128 \times 128 \times 129$ grid points at $\operatorname{Re}_{\tau}^{L}$, with $N_{x} \times N_{y} \times N_{z}=256 \times 256 \times 257$ grid points at $\operatorname{Re}_{\tau}^{I}$, and $N_{x} \times N_{y} \times N_{z}=512 \times 512 \times 513$ grid points at $\operatorname{Re}_{\tau}^{H}$. Spectral representation of flow variables uses wave numbers $k_{x}=2 \pi \mathcal{I} / L_{x}$ and $k_{y}=2 \pi \mathcal{J} / L_{y}$ (with $-N_{x} / 2+1 \leqslant \mathcal{I} \leqslant+N_{x} / 2$, and $-N_{y} / 2+1 \leqslant \mathcal{J} \leqslant+N_{y} / 2$ before dealiasing), and coefficients $T_{n}(\mathcal{Z})=\cos [n \arccos (\mathcal{Z})]$ (with $n=1, \ldots, N_{z}$ before dealiasing and $-1 \leqslant \mathcal{Z} \leqslant 1$ ).

Swimmers are tracked using a Lagrangian approach. Each swimmer is modeled as a spherical particle whose position $\mathbf{x}_{p}$ evolves according to

$$
\frac{d \mathbf{x}_{p}}{d t}=\mathbf{u}_{@ p}\left(\mathbf{x}_{p}, t\right)+v_{s} \mathbf{p},
$$

where $v_{s}$ is the (constant) swimming speed, $\mathbf{u}_{@ p}\left(\mathbf{x}_{p}, t\right)$ the velocity of fluid at the swimmer location and $\mathbf{p}$ is the unit vector that defines the spatial orientation of the swimmer. The orientation vector $\mathbf{p}$ evolves in time according to the response of the swimmer to the biasing torques acting upon it: The viscous torque on the swimmer body, caused by the local shear, and the gravitactic torque, arising from bottom heaviness [11], as shown in Fig. 2. For spherical inertialess swimmers, the orientation 
rate is computed from the following equation $[5,32]$ :

$$
\frac{d \mathbf{p}}{d t}=\frac{1}{2 \mathcal{B}}[\mathbf{k}-(\mathbf{k} \cdot \mathbf{p}) \mathbf{p}]+\frac{1}{2} \omega_{@ p} \wedge \mathbf{p},
$$

where $\mathbf{k}=[0,0,1]$ is a unit vector pointing upward in the vertical direction, $\boldsymbol{\omega}_{@ p}$ is the fluid vorticity at the swimmer position and $\mathcal{B}$ is the characteristic time a perturbed swimmer takes to restore its vertical orientation when $\omega_{@ p}=0$. Such reorientation time can be computed as $\mathcal{B}=\mu \alpha_{\perp} /(2 \mathcal{H} \rho g)$, where $\alpha_{\perp}$ is the dimensionless resistance coefficient for rotation about an axis perpendicular to $\mathbf{p}$ and $\mathcal{H}$ is the center-of-mass offset relative to the center of buoyancy (located at point B in Fig. 2). The first term on the right-hand side of Eq. (4) describes the tendency of a swimmer to remain aligned along the vertical direction due to bottom-heaviness, while the second term describes the tendency of fluid vorticity to overturn the swimmer by imposing a viscous torque on it. A possible variation to Eq. (4) may stem from the inclusion of the effect of the acceleration induced by the fluid along the cell trajectory [17,33]. This effect has been found to generate multifractal plankton clustering in the high vorticity regions of the flow for the case of homogeneous isotropic turbulence [17]. The relative importance of gravitational acceleration $\mathbf{g}$ and fluid acceleration a on plankton dynamics depends on the ratio $g / a_{\text {RMS }}$ where $a_{\text {RMS }}$ is the root-mean square (RMS) of a [17,33]. For flows at low or moderate Reynolds number, namely, $\operatorname{Re}_{\tau}^{L}$ and $\operatorname{Re}_{\tau}^{I}$ in the present study, $g / a_{\mathrm{RMS}} \gg 1$ (especially at high stability numbers, as discussed in Ref. [33]) and, therefore, the neglect of $\mathbf{a}$ in Eq. (4) appears fully justified. Some effect might be expected at high $\operatorname{Re}_{\tau}$ upon consideration of the characteristic acceleration scales in the different simulations: In outer units, the acceleration scale is $U_{\max }^{2} / h$ where $U_{\max }$ is the fluid velocity at the free surface, whereas the acceleration scale in viscous units is $u_{\tau}^{3} / \nu$. Based on these definitions, the dimensional values of the acceleration scale in outer units are $0.03,0.4374$, and $2.16 \mathrm{~m} \mathrm{~s}^{-2}$ for $\operatorname{Re}_{\tau}^{L}, \operatorname{Re}_{\tau}^{I}$, and $\operatorname{Re}_{\tau}^{H}$, respectively, while the values in viscous units are $0.027,0.729$, and $5.83 \mathrm{~m} \mathrm{~s}^{-2}$. Comparison with $g=9.81 \mathrm{~ms}^{-2}$ indicates that the fluid acceleration term might be of importance only for the $\operatorname{Re}_{\tau}^{H}$ case with low stability number. Even for this case, however, we decided to use Eq. (4), considering that the condition $g / a_{\text {RMS }} \gg 1$ is typical of most marine environments, where cell distributions are weakly affected by fluid acceleration effects [17]. The choice is also motivated by the fact that the gyrotactic swimmers considered in this study tend to avoid the bottom boundary of the channel, where inhomogeneous flow conditions could generate high vorticity and drive fluid-acceleration-induced clustering. Another simplification in Eq. (4) is the neglect of rotational diffusivity, which is typically associated with Brownian motion or other cell-scale random motions. Yet, the size of most algal cells (and of those considered in this study, in particular) is large enough for Brownian motions to be deemed negligible [5].

Based on the above considerations, the position and swimming direction of each swimmer were integrated in time using the nondimensional form of Eqs. (3) and (4):

$$
\begin{gathered}
\frac{d \mathbf{x}_{p}^{+}}{d t^{+}}=\mathbf{u}^{+}\left(\mathbf{x}_{@ p}^{+}\right)+\Phi^{+} \mathbf{p}, \\
\frac{d \mathbf{p}}{d t^{+}}=\Psi^{+}[\mathbf{k}-(\mathbf{k} \cdot \mathbf{p}) \mathbf{p}]+\frac{1}{2} \omega^{+} @ p \wedge \mathbf{p},
\end{gathered}
$$

where time, length, and velocity variables are made nondimensional using $u_{\tau}$ and $\nu$. The key parameters in Eqs. (5) and (6) are the swimming number $\Phi^{+}=v_{s} / u_{\tau}$ and the stability number $\Psi^{+}=\frac{1}{2 \mathcal{B}} \frac{v}{u_{\tau}^{2}}$, which parametrizes the importance of vortical overturning with respect to directional swimming. Time integration exploits a fourth-order Adams-Bashforth scheme, starting from a three-dimensional random distribution (in both space and orientation) of the swimmers throughout the channel. Swimmers are tracked using a point-particle approach, justified by the sub-Kolmogorov size typical of aquatic microorganisms [5], and are injected into the flow at a concentration low enough to neglect the effect of the swimmers on turbulence (one-way coupling) and the effect of possible collisions between swimmers. Present results, therefore, apply only to dilute flow 
conditions. The fluid velocity and vorticity at the instantaneous location of the swimmer is obtained through interpolation based on sixth-order Lagrange polynomials. Periodic boundary conditions are imposed on swimmers moving outside the computational domain in the horizontal (homogeneous) directions. In the wall-normal direction, the swimmers rebound elastically at both boundaries: For a detailed discussion on the assumption of fully elastic rebound to describe swimmer interaction with a free surface, the reader is referred to Mashayekpour et al. [20].

Samples of $\mathcal{N}=10^{6}$ swimmers with density ratio $S=\rho_{p} / \rho_{f}=1$ and diameter $d_{p}=250 \mu \mathrm{m}$ (a value in the size range of large phytoplankton cells) $[4,13]$ were considered. Since we focus on inertialess swimmers, added mass effects can be safely disregarded [14-17,21-23]. The value of $\mathcal{N}$ was chosen to ensure statistical convergence. The corresponding mass and volume fractions are $\Phi_{m}=\Phi_{V}=2.28 \times 10^{-3}$, low enough to justify the assumption of dilute flow in the bulk of the flow.

For each sample, three different values of the stability number were considered: $\Psi_{L}^{+}=0.0113$, $\Psi_{I}^{+}=0.113$, and $\Psi_{H}^{+}=1.13$, corresponding to low, intermediate, and high gyrotaxis, respectively. In dimensional units, these values correspond to reorientation times in the range $0.1 s<\mathcal{B}<10 s$, which is also typical of motile phytoplankton species [4,13] (in particular, we have $\mathcal{B} \simeq 0.05$, $0.5,5.0 \mathrm{~s}$ at $\operatorname{Re}_{\tau}^{L}$ ). A fixed value of the swimming velocity $\Phi^{+}=0.048$ is used, corresponding to $144 \mu \mathrm{m} / \mathrm{s}$ at $\operatorname{Re}_{\tau}^{L}$ and $864 \mu \mathrm{m} / \mathrm{s}$ at $\operatorname{Re}_{\tau}^{H}$ : these values fall within the range $10 \mu \mathrm{m} / \mathrm{s}<\mathrm{v}_{\mathrm{s}}<$ $1000 \mu \mathrm{m} / \mathrm{s}$, also typical of motile phytoplankton [4,13], and yield a constant surfacing time, defined as the time taken by a swimmer to cover the whole channel height, among the different simulations. In summary, a total of 9 cases in the $\left(\operatorname{Re}_{\tau}, \Psi^{+}\right)$parameter space was considered.

\section{RESULTS AND DISCUSSION}

In this section, we will first characterize the flow field in terms of velocity statistics and energy spectra to show the separation of scales acting on the swimmers at the different Reynolds numbers. Then, we will examine the free-surface clustering of the swimmers and the surfacing dynamics that lead to cluster formation. The analysis will focus on flow scale effects for swimmers with different gyrotaxis.

\section{A. Flow field characterization}

We start our analysis by considering the mean and root-mean square (RMS) fluid velocity profiles for the three Reynolds numbers, shown in Fig. 3 as a function of the wall-normal coordinate $z^{+} / \operatorname{Re}_{\tau}$ (equal to zero at the bottom wall and equal to unity at the free surface). Brackets indicate time and space average (over the homogeneous flow directions). The averaging procedure is the same adopted in Lovecchio et al. [24,30]. Our results are in good agreement with those reported in previous studies [26], for the two lower values of $\mathrm{Re}_{\tau}$ (comparison not shown for sake of brevity). As expected when using wall units, velocity profiles shown in Fig. 3(a) overlap perfectly in the near-wall region regardless of the Reynolds number. We observe also that the free surface does not alter significantly the mean velocity, which follows the logarithmic profile throughout the outer flow layer except in the fluid sublayer attached to the free surface. In such layer, the strong effect of the surface is revealed by the nonvanishing streamwise and spanwise components of the RMS, shown in Figs. 3(b)-3(d), which indicate the presence of an anisotropic velocity region [26,28]. Finally, we note that, compared to the channel height, the thickness of the viscous sublayer attached to the bottom wall reduces significantly as $\mathrm{Re}_{\tau}$ increases, indicating a marked separation with respect to the upper surface-influenced region and weaker turbulence anisotropy.

The statistical moments shown in Fig. 3 do not provide direct information about the turbulent flow structures near the free surface [26,27,34-38], which are known to produce a flow with properties that differ from those typical of two-dimensional incompressible Navier-Stokes turbulence $[39,40]$. Here, we examine these properties by discussing the energy spectra of the fluid velocity fluctuations on the surface at statistically-steady state [26], shown in Fig. 4 for each Reynolds 

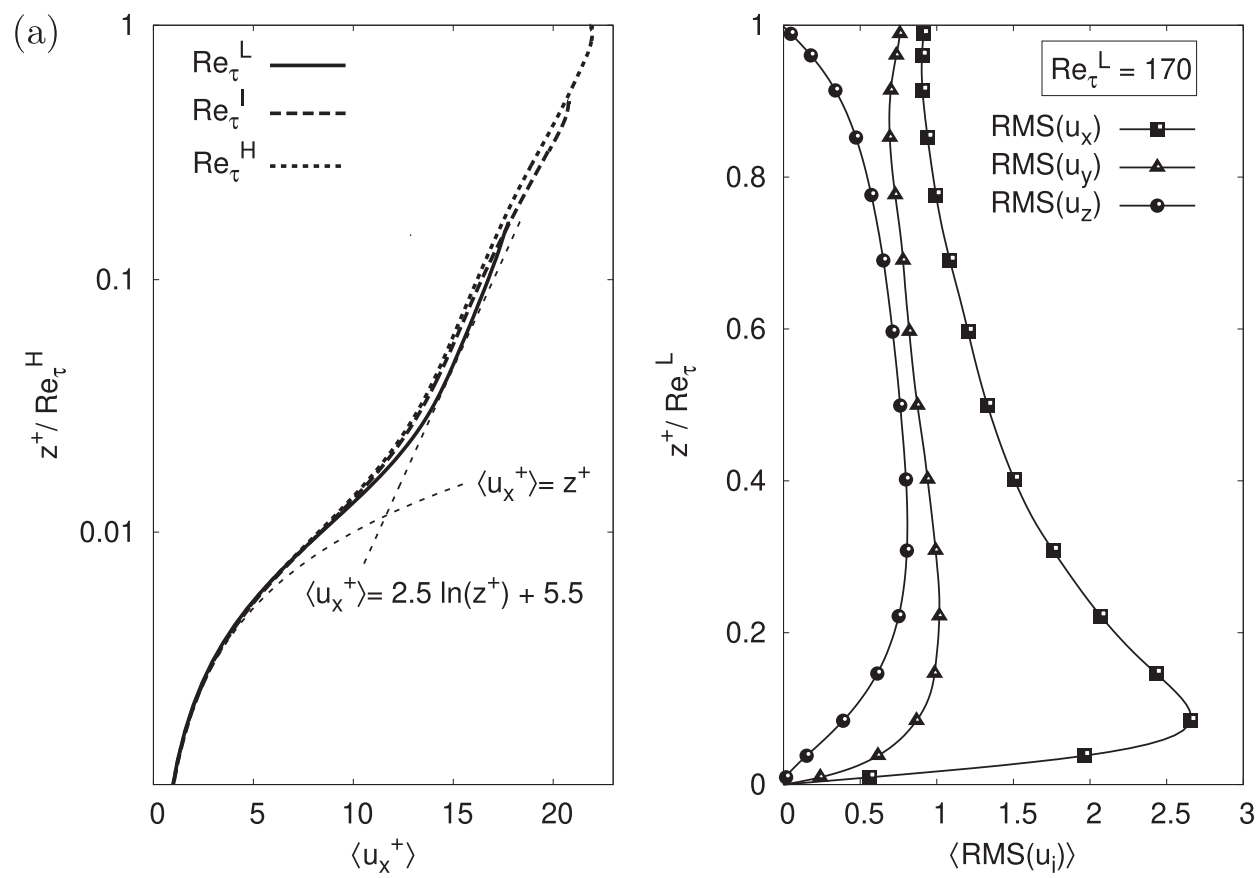

(b)
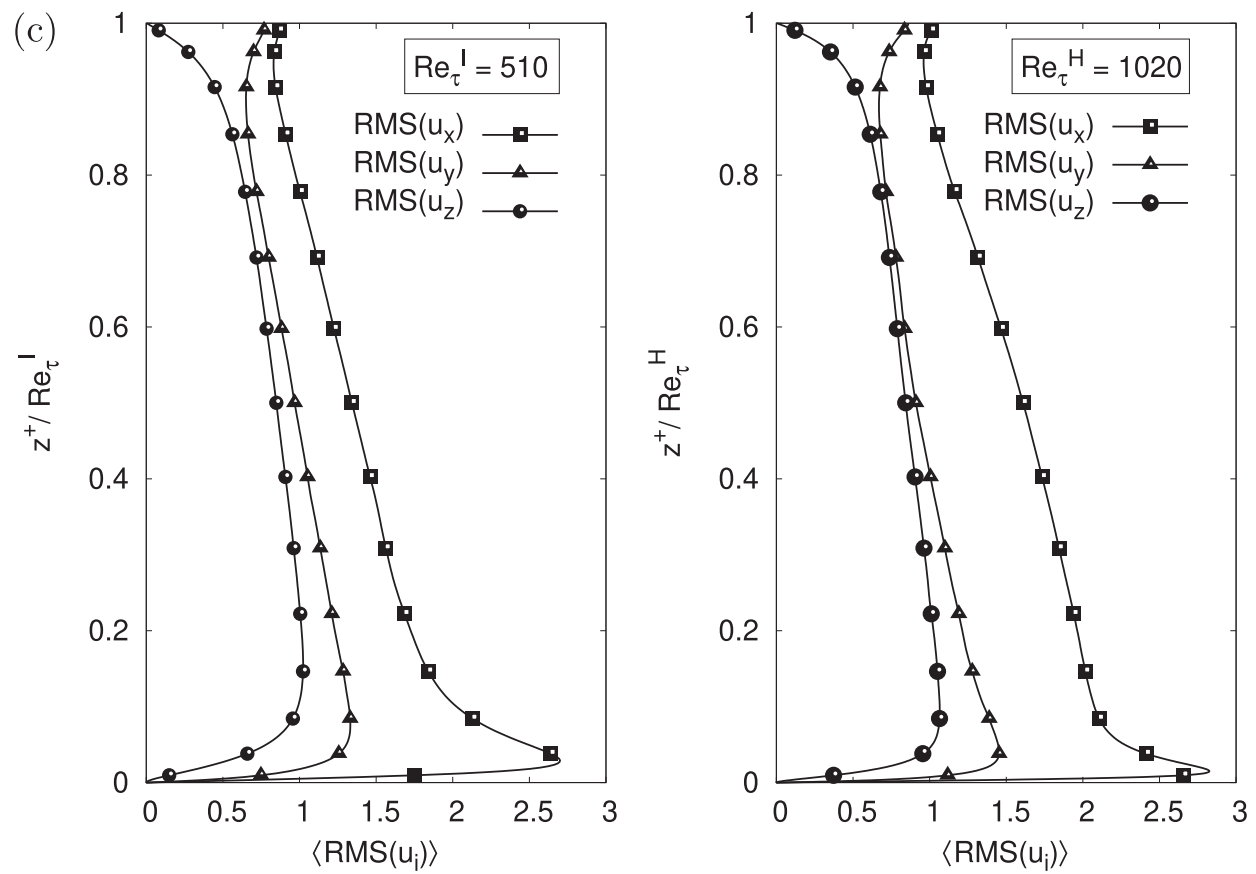

(d)

FIG. 3. Fluid velocity statistics. Panels: (a) mean velocity profiles for all Reynolds numbers; (b) fluid velocity $\mathrm{RMS}$ at $\operatorname{Re}_{\tau}^{L}=170$; (b) fluid velocity $\mathrm{RMS}$ at $\operatorname{Re}_{\tau}^{I}=510$; (c) fluid velocity $\mathrm{RMS}$ at $\operatorname{Re}_{\tau}^{H}=1020$.

number. We remark here that, in the present flow configuration, the Reynolds number can be interpreted as the dimensionless depth of the channel and, hence, as a scaling parameter for the large eddies of the flow. To emphasize direction-related aspects of the energy spectra, results 

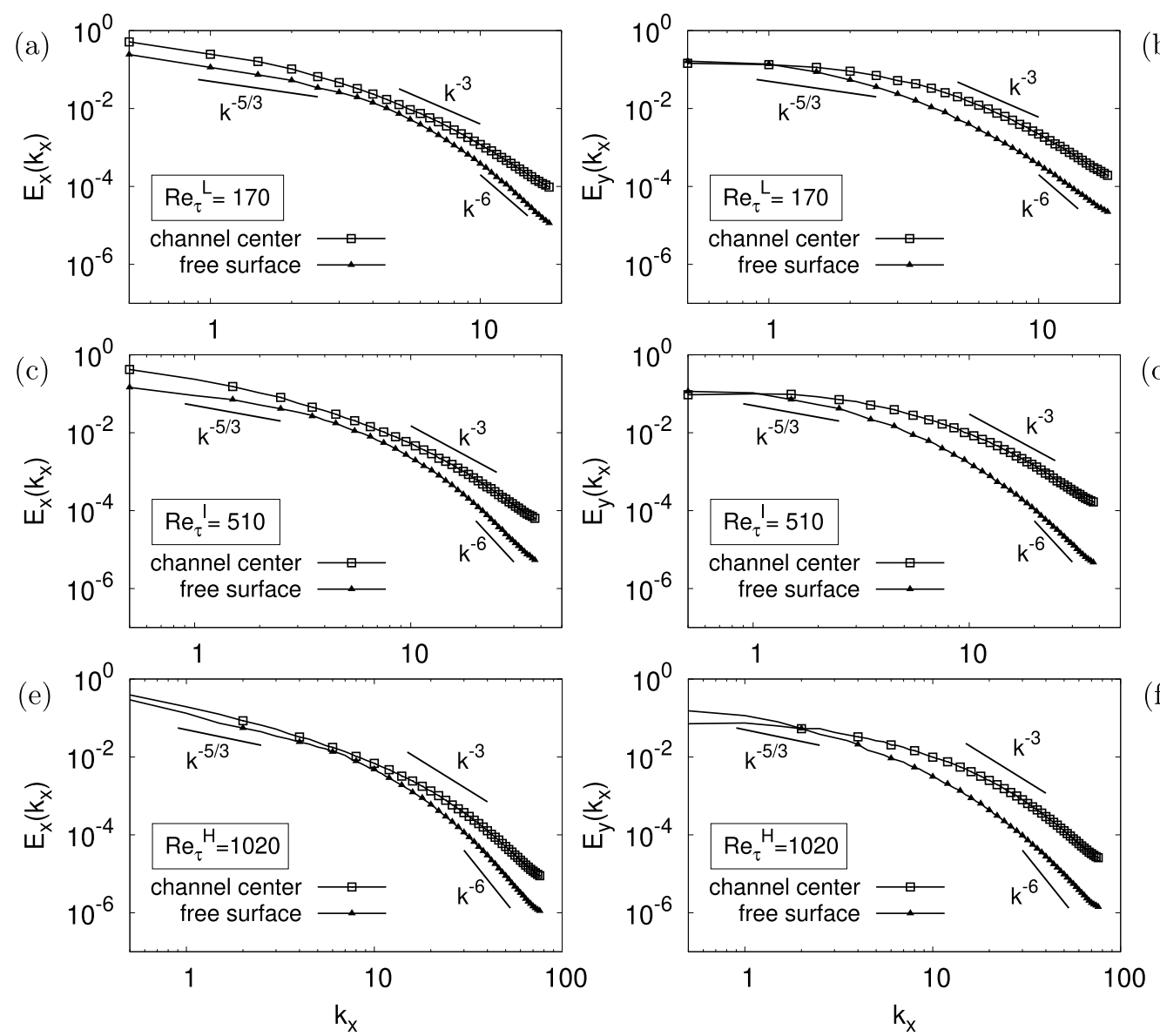

FIG. 4. One-dimensional (streamwise) energy spectra of the streamwise and spanwise surface-parallel velocity fluctuations, $E_{x}\left(k_{x}\right)$ (left-hand panels), and $E_{y}\left(k_{x}\right)$ (right-hand panels), respectively. Panels: (a), (b) $\operatorname{Re}_{\tau}^{L}=170$; (c), (d) $\operatorname{Re}_{\tau}^{I}=510$; (e), (f) $\operatorname{Re}_{\tau}^{H}=1020$.

for the surface-parallel velocities are examined in isolation: Figs. 4(a), 4(c) and 4(e) show the one-dimensional streamwise spectra of the streamwise velocity $E_{x}\left(k_{x}\right)$ computed at the free surface (triangles) and at the channel center (located at $z^{+}=510$ for $\operatorname{Re}_{\tau}^{H}, z^{+}=255$ for $\operatorname{Re}_{\tau}^{I}$ and $z^{+}=85$ for $\operatorname{Re}_{\tau}^{L}$, squares); Figs. 4(b), 4(d) and 4(f) show the spectra of the spanwise velocity $E_{y}\left(k_{x}\right)$ in the same two regions. Solid lines represent the slope of the spectrum within the inertial regimes predicted by the Kraichnan-Leith-Batchelor (KLB) phenomenology of two-dimensional turbulence [41,42]: $k_{x}^{-5 / 3}$, representing inverse cascade of energy to large flow scales, and $k_{x}^{-3}$, representing direct cascade of enstrophy to small flow scales. A collective analysis of the spectra shown in Fig. 4 reveals clear deviations from two-dimensionality. First, no evident $-5 / 3$ range is observed except for few of the lowest wave numbers: This can be attributed to the intermittent nature of turbulence associated with spatial fluctuations in the rate of energy dissipation. A relatively larger range of high wave numbers can be identified over which spectra exhibit a -3 scaling: In the present flow configuration, however, this corresponds to up-cascading of energy from large to small wave numbers, namely, to merging of smaller flow structures into larger structures [29]. Such findings cannot be reconciled with the KLB theory for 2D turbulence.

Examining $E_{x}\left(k_{x}\right)$, we notice that the spectrum at the free surface is always below that in the center of the channel, even if the gap between the profiles narrows as $\operatorname{Re}_{\tau}$ increases. Also, energy 
in the high-wave-number portion of the spectrum decays more rapidly, roughly as $k^{-6}$ [26]: This tendency is particularly evident at $\operatorname{Re}_{\tau}^{H}$ and indicates that only large-scale surface structures survive to the detriment of small-scale ones.

Examining $E_{y}\left(k_{x}\right)$, we observe that redistribution of energy from small to large scales in proximity of the free surface determines a cross-over between spectra at low wave numbers, when the spectrum at the free surface follows more closely the $-5 / 3$ scaling: This behavior, which becomes more evident as $\operatorname{Re}_{\tau}$ increases, is in agreement with the fact that small-scale structures become less and less important in determining turbulence properties in this region of the flow [24].

\section{B. Reynolds number and stability number effects on surface clustering}

The most evident macroscopic manifestation of floaters and motile particle dynamics in freesurface turbulence is the formation of highly concentrated clusters near the surface [13,20,24,29]. These originate from the interaction between individual cells and surface flow structures. In this section, we examine the clustering of the gyrotactic swimmers and we provide a first account of Reynolds number effects.

Surface structures are generated and sustained by near-wall bursting that generates fluid motions away from the bottom and then parallel to the free surface (upwellings) alternated to sinks associated with fluid downdrafts from the surface to the bulk (downwellings). Through these sources, fluid elements at the surface are replaced with fluid from the bulk, giving rise to the well-known surfacerenewal events [36]. To characterize the surface topology, we use the surface divergence:

$$
\nabla_{2 \mathrm{D}}=\frac{\partial u_{x}}{\partial x}+\frac{\partial u_{y}}{\partial y}=-\frac{\partial u_{z}}{\partial z}
$$

In open-channel flow, $\nabla_{2 \mathrm{D}}$ does not vanish and swimmers located on the free surface live in a compressible two-dimensional system [43], where upwellings generate regions of local flow expansion $\left(\nabla_{2 \mathrm{D}}>0\right)$ while downwellings generate regions of local compression $\left(\nabla_{2 \mathrm{D}}<0\right)$. Figure 5 provides a qualitative characterization of swimmer distribution in the upwelling and downwelling regions on the free surface by correlating the instantaneous particle patterns with the colormap of $\nabla_{2 \mathrm{D}}$. The snapshots are all taken at time $t^{+}=4000$ and refer to the same initial distribution of the swimmers. Due to gyrotaxis, swimmers can not leave the free surface by simply following flow motions: They can only leave velocity sources (red areas in Fig. 5) and collect into velocity sinks (blue areas in Fig. 5), where they organize themselves in clusters. As shown by Lovecchio et al. [24], such clusters are advected passively by the mean flow until a subsequent burst hits particles in the cluster causing its reshaping within the free surface. Eventually sharp filamentary patches of high swimmer density distribution are produced, which correlate very well with the rapidly-changing patches of $\nabla_{2 \mathrm{D}}$, as shown by Fig. 5. Formation of filamentary clusters with fractal mass distribution has been observed previously for the case of Lagrangian tracers in surface flow turbulence without mean shear $[43,44]$, with wind-induced shear [20], and for the case of floaters in free-surface turbulence [24].

A natural way to characterize surface clusters is to compute their fractal dimension, also referred to as correlation dimension $[45,46]$, which provides a quantitative measure of cluster topology. This observable was examined experimentally by Larkin et al. [44,45] and numerically by Lovecchio et al. [24] to determine the fractal dimension of the clusters formed by passive buoyant floaters in free-surface turbulence. In its three-dimensional formulation, required to study anisotropic wall-bounded flows, the correlation dimension can be computed by choosing one base particle and counting the fraction $n_{p}(r)$ of particles within a sphere of radius $r$ centered on the base particle. The correlation dimension $D_{2}$ is defined as the slope of $n_{p}(r)$ as a function of $r$ in a log-log plot. The probability distribution of the distance between the neighboring particles and the base particle is obtained by repeating this count for all possible values of $r$, thus removing any dependence on the length scale used. To compute results that are significant from a statistical point of view, in this study we repeated the procedure for many randomly-chosen base particles, averaging the results. In general, $n_{p}(r)$ scales with $r^{D_{2}}$ such that smaller values of $D_{2}$ indicate stronger preferential 
(a)
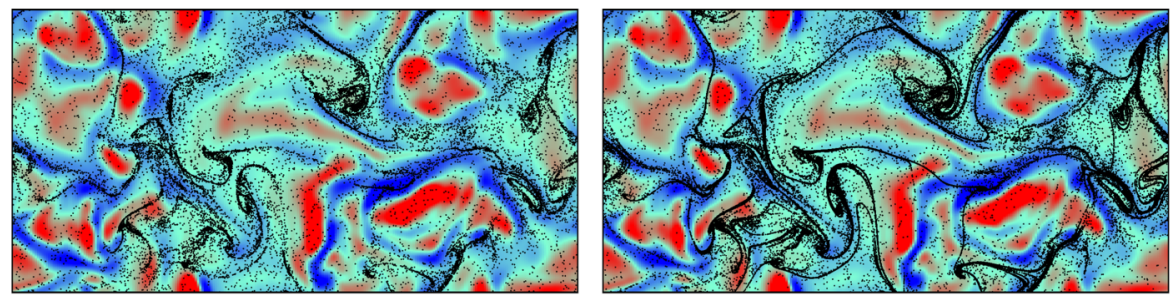

(b)

(c)
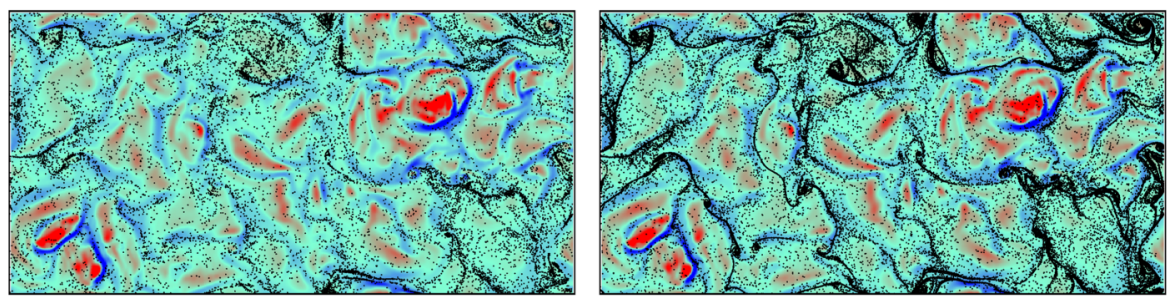

(d) $\nabla_{2 \mathrm{D}}$

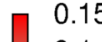

0.1

0.05

0

$-0.05$

$-0.1$

(e)

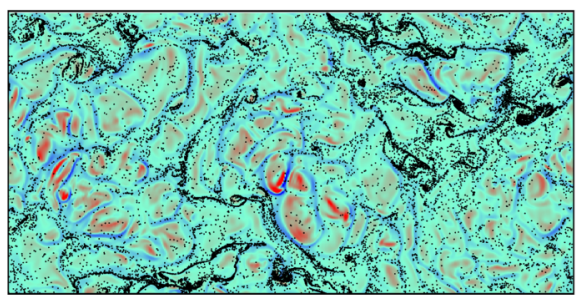

Mean Flow $\rightarrow$

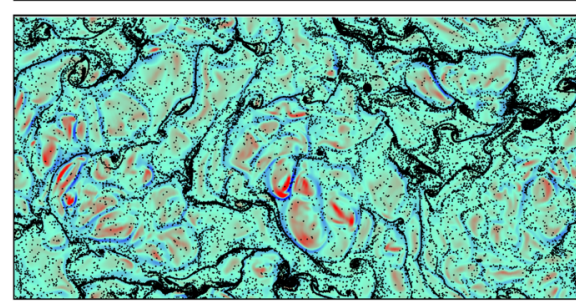

(f)

Mean Flow $\rightarrow$

FIG. 5. Top view of gyrotactic microswimmers distribution on the free surface of a turbulent open-channel flow. Flow is from left to right. Panels: (a), (b) $\operatorname{Re}_{\tau}^{L}=170$; (c), (d) $\operatorname{Re}_{\tau}^{I}=510$; (e), (f) $\operatorname{Re}_{\tau}^{H}=1020$. Left-hand panels: Low gyrotaxis, $\Psi_{L}^{+}$; right-hand panels: High gyrotaxis, $\Psi_{H}^{+}$.

concentration and segregation. In particular, if particles are uniformly distributed in the volume surrounding the base particle, then $n_{p}(r)$ scales with $r^{3}$, namely, with the volume of the sphere centered on the base particle, and $D_{2}=3$; If particles are uniformly distributed over a surface surrounding the base particle, then $n_{p}(r)$ scales with $r^{2}$, namely, with the area of the circle centered on the base particle, and $D_{2}=2$; conversely, if particles are distributed along a line centered on the base particle, then $n_{p}(r)$ scales with $r$ and $D_{2}=1$.

In Fig. 6, we show the time behavior of the correlation dimension, $D_{2}(t)$, for all Reynolds numbers and stability numbers considered in the study. It can be seen that all profiles start from $D_{2}(t=0) \simeq 3$ because of the random swimmer injection throughout the whole flow domain imposed as initial condition. As time progresses and surfacing takes place, the value of $D_{2}$ decreases significantly for the simulations with intermediate and high gyrotaxis, reaching a value slightly below unity. This trend is observed regardless of the flow Reynolds number and indicates that long-term clusters are formed at the surface (rather than in the bulk of the flow) by swimmers that are distributed along a line. More precisely, the value slightly below unity indicates that swimmers within each filament are not uniformly distributed, but rather partially overlapping (a condition possible as we neglect inter-particle collisions in the present one-way coupled simulations). We can also observe that, for the cases $\Psi_{I}^{+}$and $\Psi_{H}^{+}, D_{2}(t)$ decays at nearly the same exponential rate, with a decay time of several surface eddy turnover times (defined as the typical time for the largest eddies to significantly distort in a turbulent flow). These considerations confirm the findings of Lovecchio et al. [24], who observed that surface clusters are characterized by long-time persistence, however they do not apply to the swimmers with low gyrotaxis. The weak vertical stability of such swimmers hinders surfacing and prevents the formation of densely populated one-dimensional clusters. Indeed, the value of $D_{2}(t)$ for $\Psi_{L}^{+}$is affected by the large proportion of swimmers that remain uniformly 

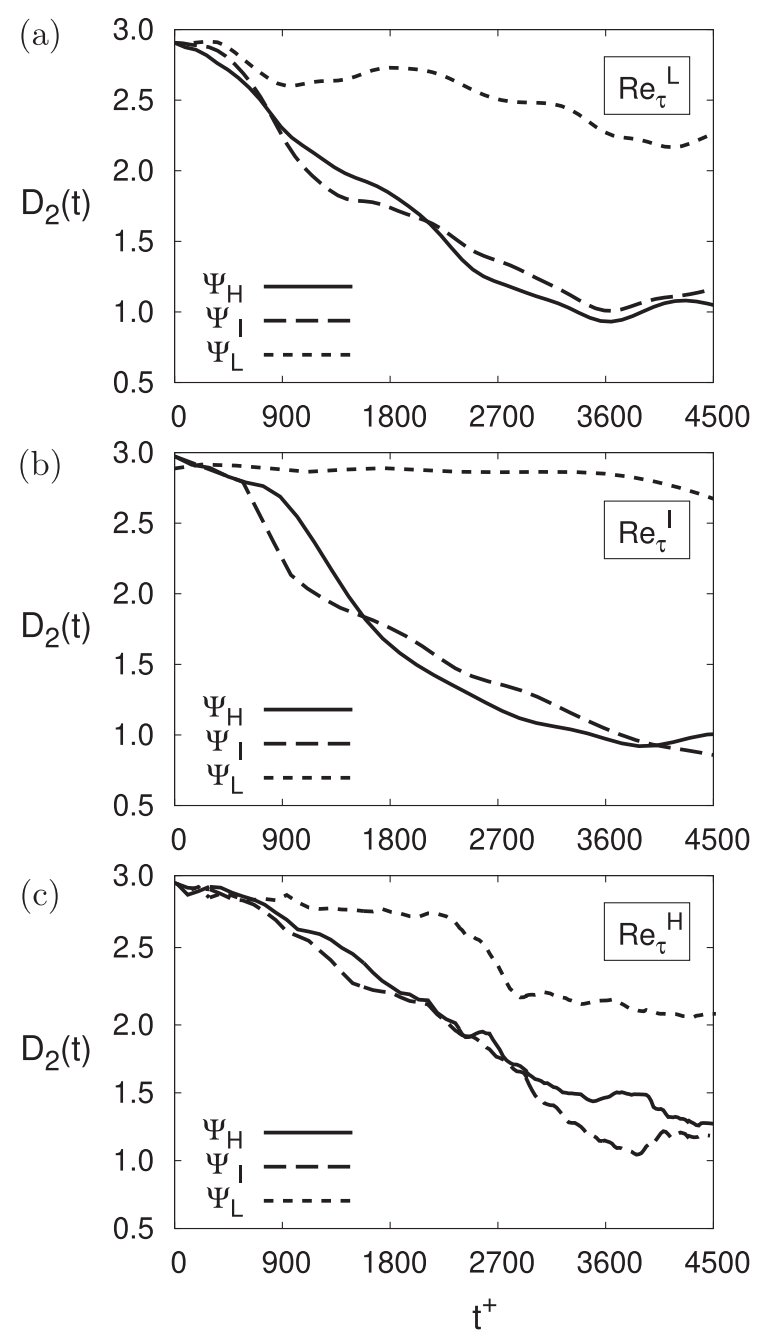

FIG. 6. Time evolution of the swimmers' correlation dimension at varying gyrotaxis and for different Reynolds numbers: (a) $\operatorname{Re}_{\tau}^{L}=170$; (b) $\operatorname{Re}_{\tau}^{I}=510$; (c) $\operatorname{Re}_{\tau}^{H}=1020$. Note that superscript + in the stability number has been dropped for ease of notation.

distributed, being still located in the bulk of the flow, where gyrotaxis alone is not able to induce significant preferential concentration in the absence of inertia.

The results just discussed highlight already the presence of significant Reynolds number effects on the swimmer dynamics, and hint to possible saturation effects on vertical migration efficiency above a certain level of vertical stability. These aspects of the problem are further analyzed in the next section, with specific reference to the rising of the swimmers toward the free surface.

\section{Influence of turbulent flow scales and gyrotaxis on surfacing}

One feature of swimmer dynamics that is clearly highlighted by Fig. 5, is the different number of cells that have reached the surface at the time instant of the visualizations. Swimmers with low gyrotaxis (low stability number $\Psi_{L}^{+}$, left-hand panels), are out-numbered by swimmers with high gyrotaxis (high stability number $\Psi_{H}^{+}$, right-hand panels). To quantify this qualitative observation, in panels (a) of Figs. $7-9$ we show the wall-normal concentration profiles, $C / C_{0}$, computed at the 

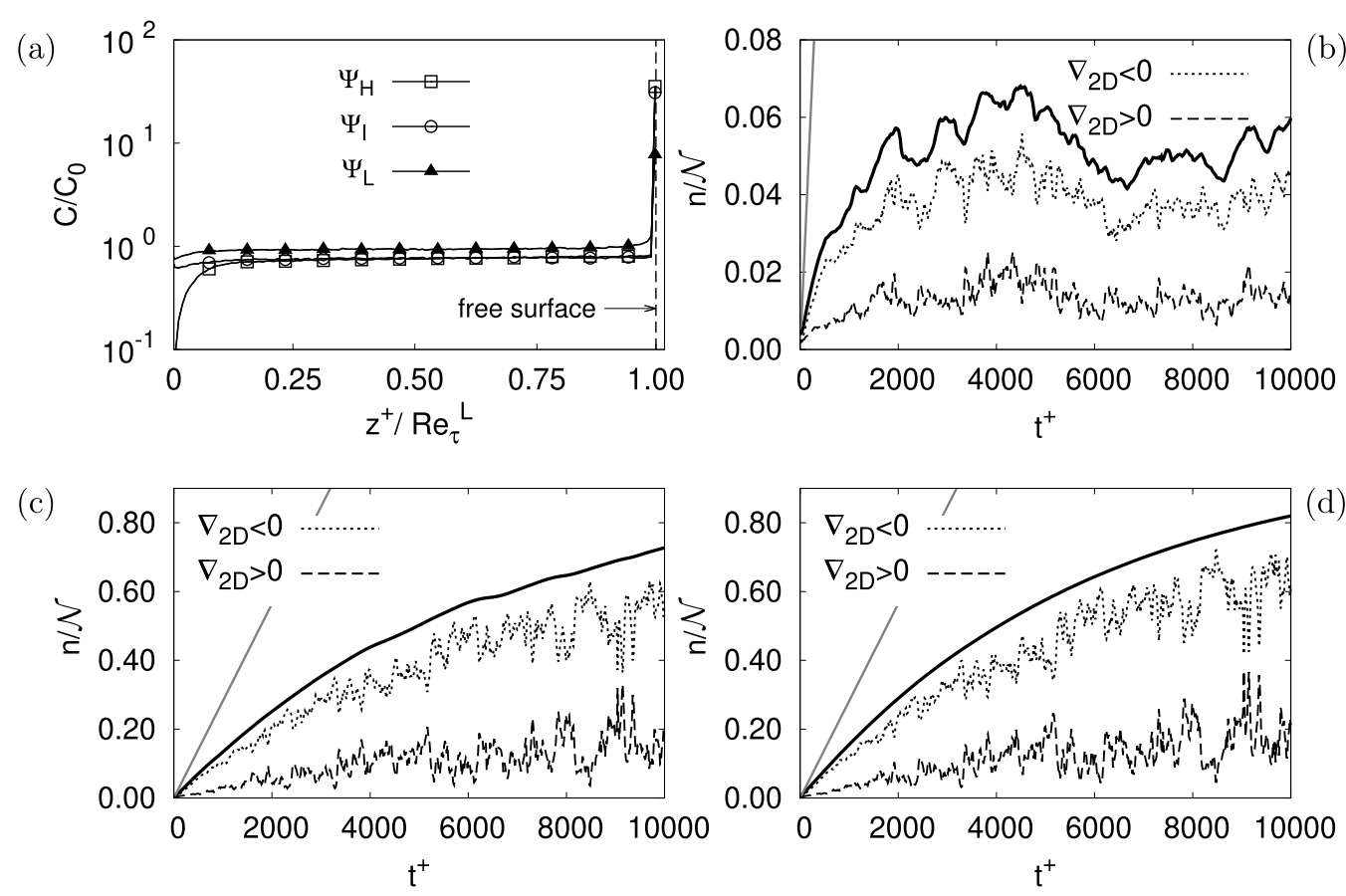

FIG. 7. Surfacing and accumulation of gyrotactic microswimmers $\left(\operatorname{Re}_{\tau}^{L}=170\right)$. Panels: (a) Wall-normal concentration at varying gyrotaxis in log-linear scale; (b), (c), (d) Evolution of the number of swimmers that have reached the subsurface layer $1<\left(\operatorname{Re}_{\tau}-z^{+}\right) / d_{p}^{+}<5$ for low, intermediate, and high gyrotaxis. The relative contributions due to swimmers in downwellings $\left(\nabla_{2 \mathrm{D}}<0\right.$, dash-dotted lines) and upwellings $\left(\nabla_{2 \mathrm{D}}>0\right.$, dashed lines) are also shown. In panel (a), the stability numbers are indicated without superscript + for ease of notation. In panels (b)-(d), the gray line represents the evolution of $n / \mathcal{N}$ for the case of swimmers rising in still fluid.

same time of Fig. 5, comparing results at varying gyrotaxis, each figure pertaining to a different Reynolds number. Note that the concentration, $C$, represents the instantaneous number density of swimmers per unit volume (normalised by its initial value $C_{0}=C(t=0)$, such that $C / C_{0}>1$ indicates preferential concentration and $C / C_{0}<1$ indicates depletion) and is shown as a function of the wall-normal coordinate $z^{+}$normalised by the Reynolds number of the simulation. In panels (b-d) of these figures, we show the time evolution of the number $n$ of swimmers that have reached the subsurface layer $1<\left(\operatorname{Re}_{\tau}-z^{+}\right) / d_{p}^{+}<5$ for low, intermediate and high gyrotaxis, respectively (solid lines in each panel). The location and thickness of the layer was chosen to remove the thin fluid slab within which swimmers interact with the free surface, thus minimizing the effect of the rebound condition on the statistical observable, and to ensure a sufficiently long residence time for the swimmers. The value of $n$ is normalised by the total number $\mathcal{N}$ of swimmers tracked in each simulation and its variation over time can also be interpreted as the mass flux of swimmers reaching the surface. In addition, the dashed and dotted curves in panels (b-d) represent the fraction of swimmers located in regions of the monitor subsurface layer characterized by negative and positive values of the surface divergence, respectively. Finally, the straight gray lines represent the fraction of swimmers that would have reached the free surface in the case of still fluid, namely, when $\mathbf{u}_{@ p}=0$ in Eqs. (5) and (6).

As far as concentration is concerned, we observe a buildup of concentration right at the surface for all values of $\mathrm{Re}_{\tau}$. The peak of concentration increases monotonically with gyrotaxis, indicating a stronger migration toward the free surface. Interestingly, the peak value of $C / C_{0}$ is nearly the 

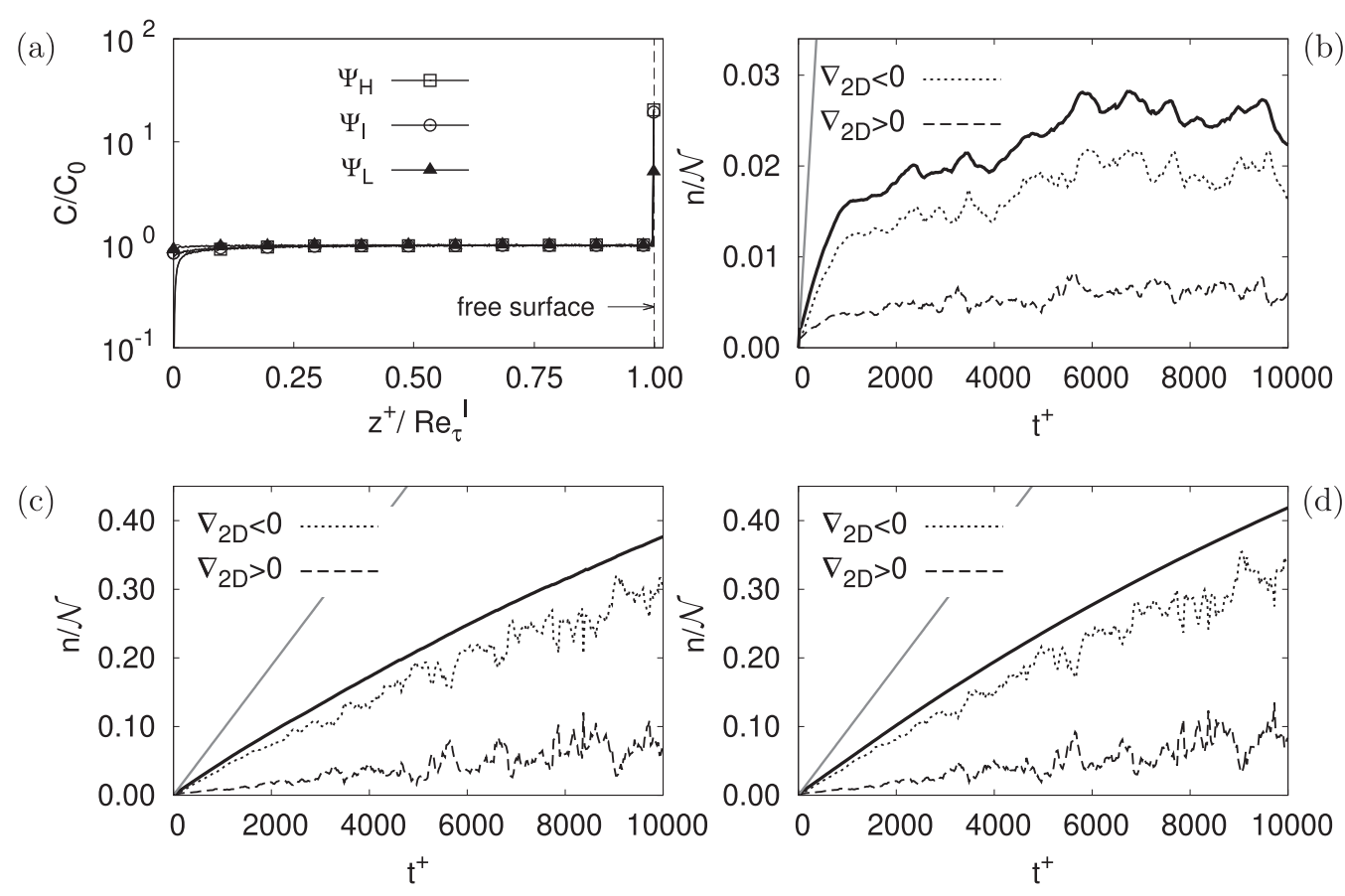

FIG. 8. Surfacing and accumulation of gyrotactic microswimmers $\left(\operatorname{Re}_{\tau}^{I}=510\right)$. Panels: (a) Wall-normal concentration at varying gyrotaxis in log-linear scale; (b), (c), (d) Evolution of the number of swimmers that have reached the subsurface layer $1<\left(\operatorname{Re}_{\tau}-z^{+}\right) / d_{p}^{+}<5$ for low, intermediate and high gyrotaxis. The relative contributions due to swimmers in downwellings $\left(\nabla_{2 \mathrm{D}}<0\right.$, dash-dotted lines) and upwellings $\left(\nabla_{2 D}>0\right.$, dashed lines) are also shown. In panel (a), the stability numbers are indicated without superscript + for ease of notation. In panels (b)-(d), the gray line represents the evolution of $n / \mathcal{N}$ for the case of swimmers rising in still fluid.

same for the cases $\Psi_{I}^{+}$and $\Psi_{H}^{+}$, confirming that the ability of the swimmers to rise upwards saturates above a certain threshold of vertical stability. In the bulk of the flow, the distribution of the swimmers nearly remains uniform, indicating absence of accumulation in horizontal layers, whereas the bottom wall is depleted of cells: This is attributed to the tendency of swimmers to preferentially concentrate in the upwelling regions of the flow when rising toward the surface and in the downwelling regions when leaving the surface, in agreement with [14,16,21]. To provide a qualitative rendering of the time evolution of the concentration at the free surface in the $\left(\operatorname{Re}_{\tau}, \Psi^{+}\right)$ space, in Fig. 10, we report the peak values of $C / C_{0}$ at two different times: $t^{+}=2000$ [Fig. 10(a)] and $t^{+}=4000$ [Fig. 10(b)]. Linear interpolation of these values produces the gray surfaces shown in the figure, which provide a visual rendering of the concentration peaks that could be expected for values of $\operatorname{Re}_{\tau}$ and $\Psi^{+}$not covered by the present study. Figure 10 shows clearly that the peak of concentration is a decreasing function of $\operatorname{Re}_{\tau}$ and an increasing function of $\Psi^{+}$.

We remark here that only the concentration profiles of the $\Psi_{L}^{+}$swimmers at the two lowest Reynolds numbers have reached the steady state within the simulated time window. This is clearly visible from the evolution of $n / \mathcal{N}$, which eventually oscillates around a mean value only in Figs. 7(b) and 8(b) while increasing steadily in all other cases. More specifically, less than $10 \%$ of the $\Psi_{L}^{+}$swimmers are able to reach the surface at $\operatorname{Re}_{\tau}=170$, this percentage being halved at $\operatorname{Re}_{\tau}=$ 510 and $\operatorname{Re}_{\tau}=1020$. For the cases of intermediate and high gyrotaxis, $n / \mathcal{N}$ reaches much higher values (up to roughly $80 \%$ at $\operatorname{Re}_{\tau}=170$ and $40 \%$ at $\operatorname{Re}_{\tau}=1020$ for the $\Psi_{H}^{+}$swimmers). These percentage values are always lower than those characterizing swimmers in still fluid (represented 

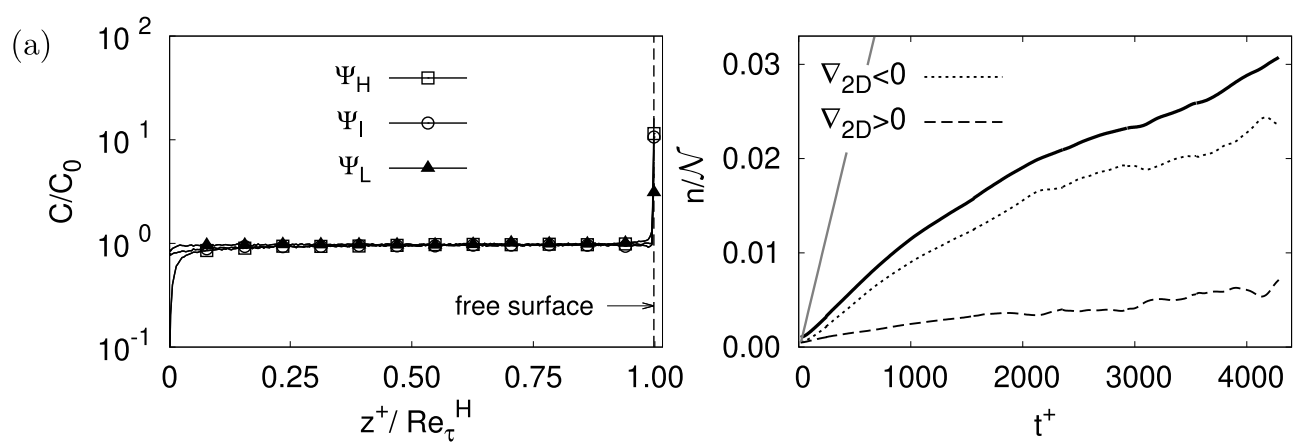

(c)
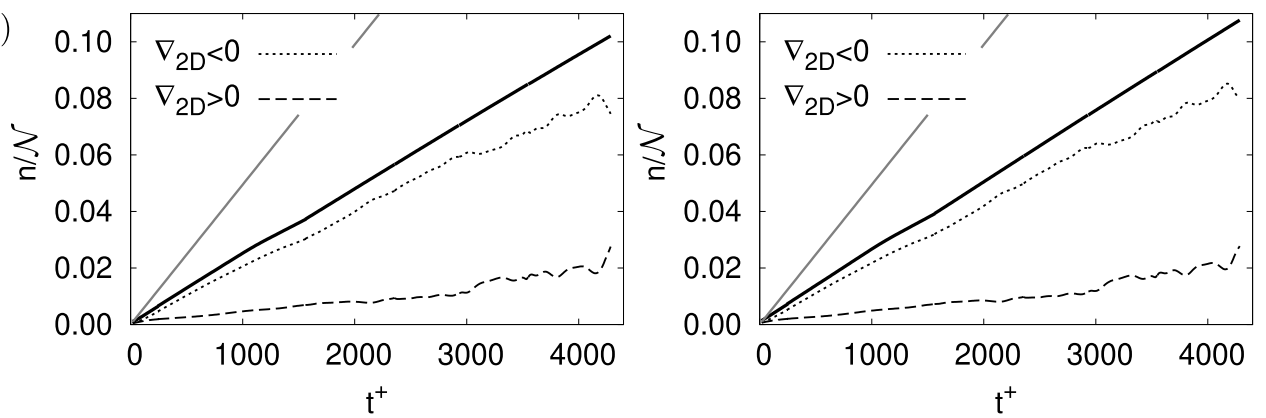

(d)

FIG. 9. Surfacing and accumulation of gyrotactic microswimmers $\left(\operatorname{Re}_{\tau}^{H}=1020\right)$. Panels: (a) Wall-normal concentration at varying gyrotaxis in log-linear scale; (b), (c), (d) Evolution of the number of swimmers that have reached the subsurface layer $1<\left(\operatorname{Re}_{\tau}-z^{+}\right) / d_{p}^{+}<5$ for low, intermediate and high gyrotaxis. The relative contributions due to swimmers in downwellings $\left(\nabla_{2 \mathrm{D}}<0\right.$, dash-dotted lines) and upwellings $\left(\nabla_{2 D}>0\right.$, dashed lines) are also shown. In panel (a), the stability numbers are indicated without superscript + for ease of notation. In panels (b)-(d), the gray line represents the evolution of $n / \mathcal{N}$ for the case of swimmers rising in still fluid.

by the gray line in each panel of Figs. 7-9). In this latter case, swimmers would rise steadily with velocity $v_{s}$ and reach the surface within a maximum time $\mathcal{T}^{+}=h^{+} / \Phi^{+}=\operatorname{Re}_{\tau} / \Phi^{+}$, with $n / \mathcal{N}=1$ at $t^{+}=\mathcal{T}^{+}\left(\mathcal{T}^{+} \simeq 3.5 \times 10^{3}\right.$ for $h^{+}=\operatorname{Re}_{\tau}^{L} ; \mathcal{T}^{+} \simeq 10.5 \times 10^{3}$ for $h^{+}=\operatorname{Re}_{\tau}^{I}$ and $\mathcal{T}^{+} \simeq 21 \times 10^{3}$
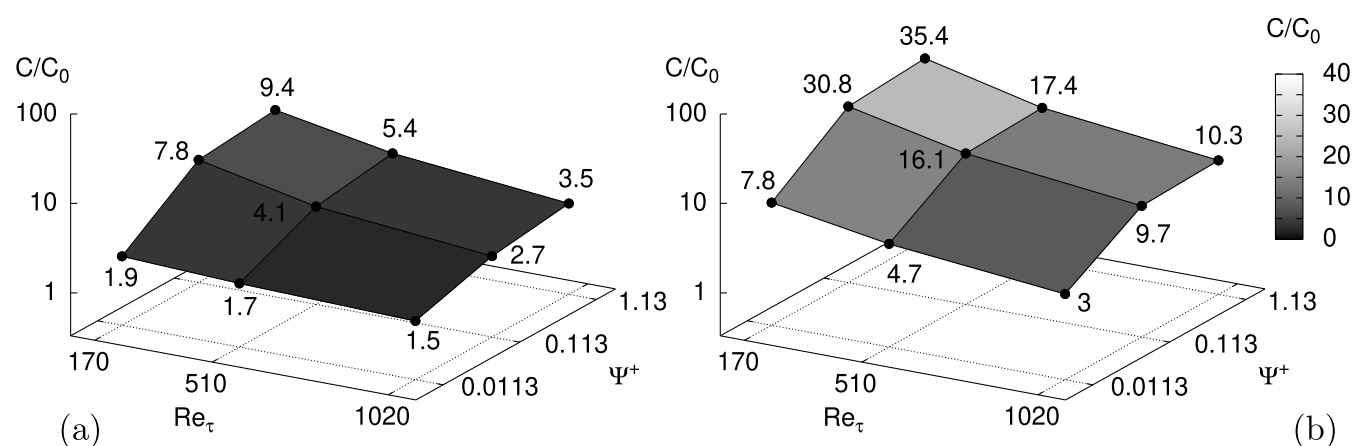

FIG. 10. Peak values of swimmer concentration $C / C_{0}$ at the free surface in the $\left(\operatorname{Re}_{\tau}, \Psi^{+}\right)$parameter space. Two time steps are considered: $t^{+}=2000$ (a) and $t^{+}=4000$ (b). The gray surfaces provide a qualitative rendering of the expected peak value of $C / C_{0}$ for values of $\operatorname{Re}_{\tau}$ and $\Psi^{+}$within the range considered in this study (as provided by linear interpolation of present concentration data). 


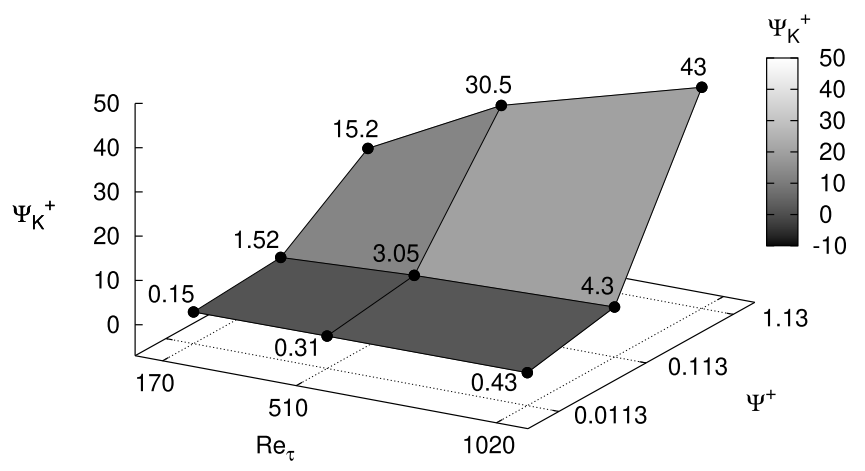

FIG. 11. Kolmogorov-based stability number, $\Psi_{K}^{+}$, in the $\left(\operatorname{Re}_{\tau}, \Psi^{+}\right)$parameter space. The gray surface provides a qualitative rendering of the expected value of $\Psi_{K}^{+}$for values of $\operatorname{Re}_{\tau}$ and $\Psi^{+}$within the range considered in this study (as provided by linear interpolation of the Kolmogorov length scales computed in this study).

for $h^{+}=\operatorname{Re}_{\tau}^{H}$ ), yet Fig. 7(c), for instance, demonstrates that only half of the high-gyrotaxis swimmers have reached the surface at $t^{+}=3.5 \times 10^{3}$ in the $\operatorname{Re}_{\tau}^{L}$ simulation. It is thus clear that turbulence and shear act to reduce the actual rise velocity of the swimmers with respect to the rise velocity in still fluid.

Figures 7-9 also indicate that the weaker turbulence anisotropy generated in the monitor layer at the two higher Reynolds numbers has a twofold effect: It smooths the $n / \mathcal{N}$ curves, and most importantly reduces by a significant amount the proportion of swimmers that reach the surface. This latter effect is a direct consequence of the decreased efficiency of near-wall bursting phenomena in bringing bulk fluid to the interface, combined with turbulence-induced destabilization of the swimmers. Finally, we note that, in agreement with the qualitative observations drawn from Fig. 5, the fraction of $n / \mathcal{N}$ that corresponds to swimmers sampling $\nabla_{2 \mathrm{D}}<0$ regions is always much higher than that corresponding to swimmers sampling $\nabla_{2 \mathrm{D}}>0$ regions. This confirms the tendency of the swimmers to accumulate continuously into downwellings when trapped near the surface.

The time evolution of $n / \mathcal{N}$ also highlights the different role played by the small and large scales of the flow in determining the pace at which swimmers can rise vertically. To discuss this role, we consider the Kolmogorov-based stability number $\Psi_{K}^{+}=\frac{1}{2 \mathcal{B}} \tau_{K}=\Psi^{+} \tau_{K}^{+}$. Dimensionless values of $\Psi_{K}^{+}$for the nine cases examined in the present study are reported in Fig. 11. Note that values refer to the maximum value of the Kolmogorov timescale in wall units, $\tau_{K \text {, max }}^{+}$, which characterizes the dissipative flow scales at the free surface [24]: More specifically, the values are $\tau_{K \text {, } m a x}^{+}=13.5,28$, and 38 at $\operatorname{Re}_{\tau}^{L}, \operatorname{Re}_{\tau}^{I}$, and $\operatorname{Re}_{\tau}^{H}$, respectively. Linear interpolation of $\Psi_{K}^{+}$values produces the gray surface shown in the figure, which provides a visual rendering of the values that could be expected for values of $\operatorname{Re}_{\tau}$ and $\Psi^{+}$not covered by the present study. For each Reynolds number, $\Psi_{K}^{+}$is smaller than one for $\Psi^{+}=\Psi_{L}^{+}$and becomes larger than unity for $\Psi^{+} \geqslant \Psi_{I}^{+}$. The case $\Psi_{K}^{+}<1$ corresponds to the situation in which the timescale of gyrotaxis is large compared to the timescale of small flow structures (especially those close to the dissipative range): Swimmers respond slowly to gravity and, therefore, cannot overcome the destabilizing influence of these small-scale structures on vertical migration. As a result, the ability of the swimmers to reach the surface through the large-scale advective structures of the flow is reduced, resulting in small values of $n / \mathcal{N}$ at the end of the simulations [see panels (a) of Figs. 7, 8, and 9].

The case $\Psi_{K}^{+}>1$ corresponds to the opposite situation in which the timescale of gyrotaxis is small compared to the timescale of the small flow structures: Swimmers' response to gravity is quick enough to counteract small-scale destabilizations on vertical migration and exploit optimally large-scale advection. This way, much higher values of $n / \mathcal{N}$ can be achieved, as shown by panels 

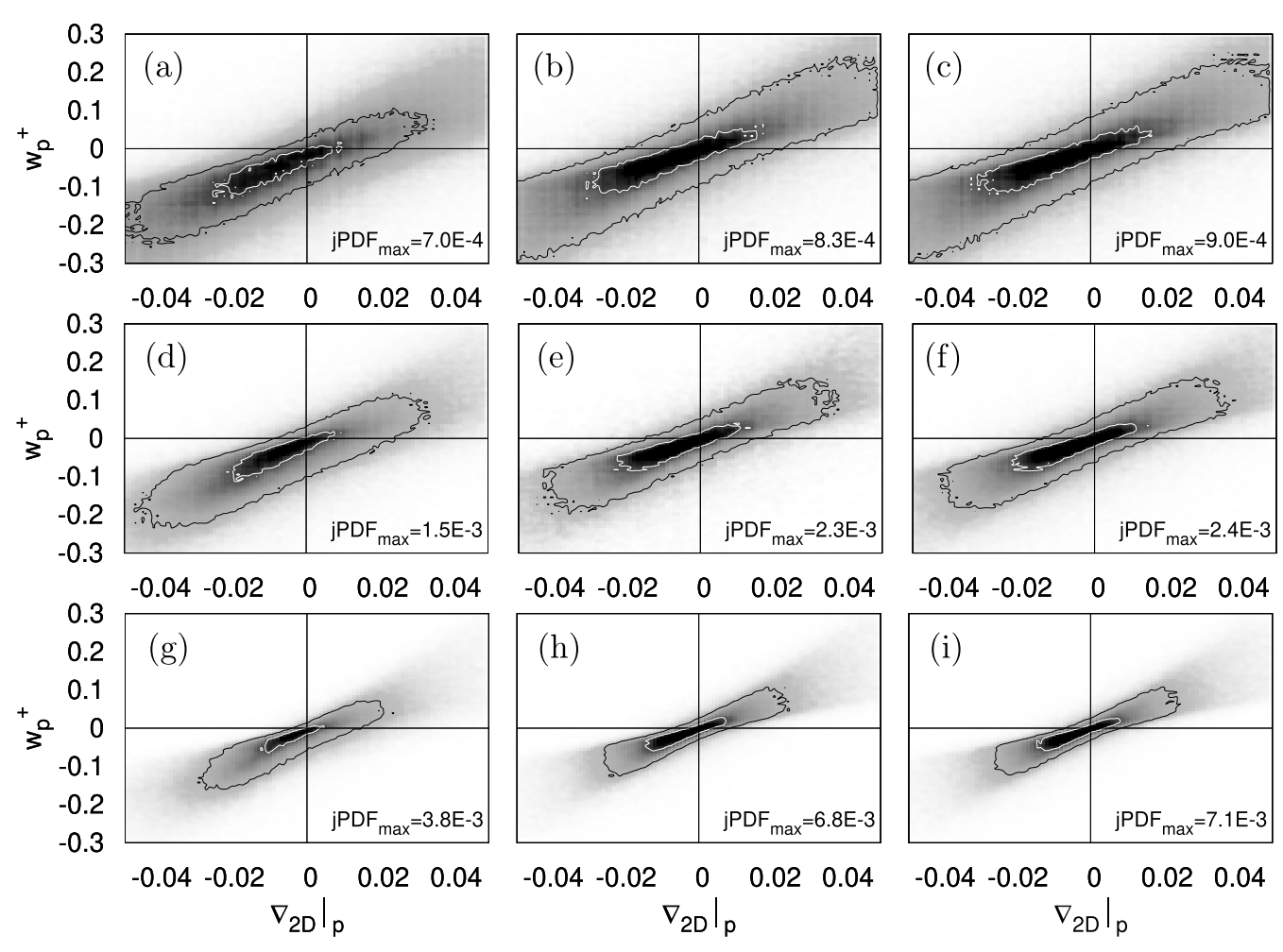

FIG. 12. Joint probability density function (jPDF) of the instantaneous vertical velocity of the swimmers, $w_{p}^{+}$, versus surface divergence, $\left.\nabla_{2 \mathrm{D}}\right|_{p}$, conditionally sampled at the swimmer positions for all three Reynolds number. Only swimmers in the region $2<z^{+}<8$ below the free surface are considered. Top-to-bottom panels: (a), (b), (c) $\operatorname{Re}_{\tau}^{L}=170$; (d), (e), (f) $\operatorname{Re}_{\tau}^{I}=510$; (g), (h), (i) $\operatorname{Re}_{\tau}^{H}=1020$. Left-to-right panels: (a), (d), (g) low gyrotaxis, $\Psi_{L}^{+}$; (b), (e), (h) intermediate gyrotaxis, $\Psi_{I}^{+}$; (c), (f), (i) high gyrotaxis, $\Psi_{H}^{+}$. White and black isocontours in each panel correspond to $50 \%$ and $10 \%$ of the maximum jPDF value.

(b) and (c) of Figs. 7, 8, and 9. Interestingly, the time behavior of $n / \mathcal{N}$ does not depend significantly on $\Psi_{K}^{+}$as long as $\Psi_{K}^{+}>1$ : This means that to ensure an efficient migration to the surface, swimmers only need the "right" amount of vertical stability to disrupt the overturning imposed by the small-scale flow structures. Based on the present results, the Kolmogorov-based stability number appears to be a suitable parameter to establish a threshold beyond which the capability of the swimmers to reach the free surface and form clusters saturates.

To conclude the analysis of surfacing dynamics, we examine in Fig. 12 the correlation between the vertical velocity of the swimmers, $w_{p}^{+}$, and the surface divergence sampled at the swimmer location, $\left.\nabla_{2 \mathrm{D}}\right|_{p}$. The correlation is evaluated by means of the joint probability density function (jPDF) of $w_{p}^{+}$and $\left.\nabla_{2 \mathrm{D}}\right|_{p}$, computed within a fluid slab $2<z^{+}<8$ below the surface (where the value of $\left.\nabla_{2 \mathrm{D}}\right|_{p}$ can be used to identify upwelling and downwelling regions) over a time window of about $500 t^{+}$. The gray-scale map shows high values of the jPDF in black (the maximum being indicated as $j P D F_{\max }$ in the right bottom corner of each panel) and low values of the jPDF in white. In each row of the figure, the Reynolds number of the flow is kept constant, and the jPDF is shown at varying gyrotaxis. To highlight the effect of increasing gyrotaxis, the colormap used in panels belonging to the same row is scaled with respect to the value of $\mathrm{jPDF}_{\max }$ calculated at $\Psi_{L}^{+}$, and isocontours correspond to $50 \%$ and $10 \%$ of such value. 
(a)

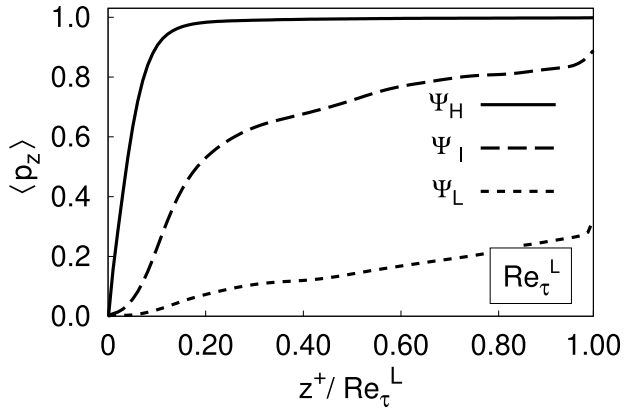

(c)

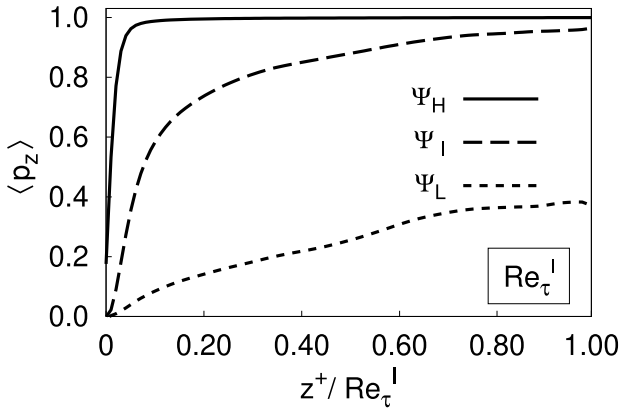

(e)

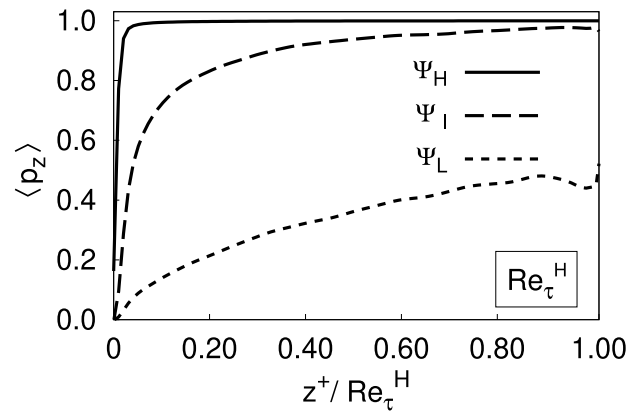

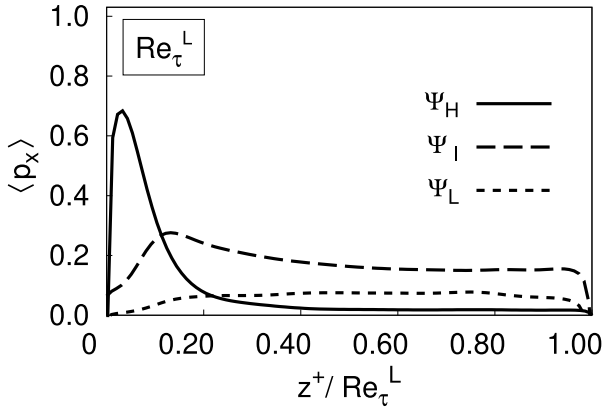

(b)

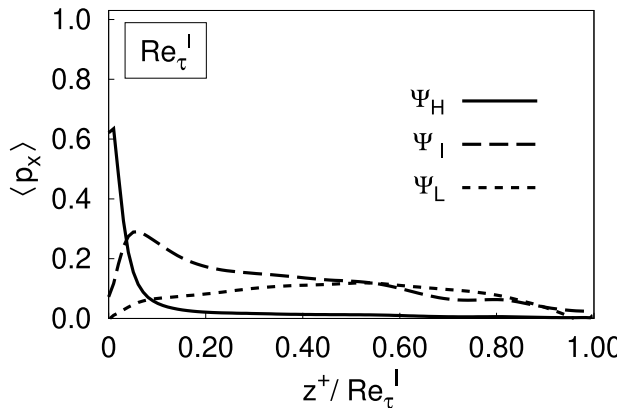

(d)

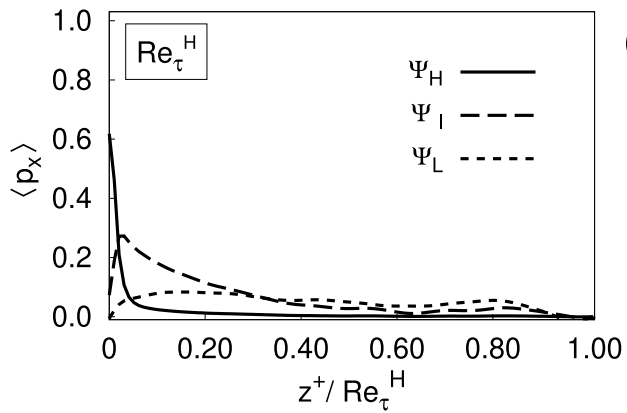

FIG. 13. Mean swimmer orientation, $\left\langle p_{i}\right\rangle$, along the wall-normal direction (left-hand panels) and along the streamwise direction (right-hand panels) at varying gyrotaxis $\left(\Psi_{L}^{+}\right.$: dotted line; $\Psi_{I}^{+}$: dashed line; $\Psi_{H}^{+}$: solid line). The free surface is located at $z^{+} / \operatorname{Re}_{\tau}=1$. Top panels: $\operatorname{Re}_{\tau}^{L}=170$; middle panels: $\operatorname{Re}_{\tau}^{I}=510$; bottom panels: $\operatorname{Re}_{\tau}^{H}=1020$.

A global inspection of Fig. 12 shows that the shape of the jPDF is similar among the cases we simulated, indicating that most of the swimmers in the selected subsurface layer either rise within upwelling regions ( $I$ quadrant events) or sink within downwelling regions (III quadrant events).

At low Reynolds number [Figs. 12(a)-12(c)], III quadrant events are characterized by the highest probability (represented by the dark gray/black areas), and the increase of vertical stability is found to favor $I$ and $I I I$ quadrant events, which are promoted by the large-scale advective motions of the flow): As $\Psi$ increases, tend to rise in upwellings and sink in downwellings. Conversely, $I I$ and $I V$ quadrant events, corresponding to swimmers rising in downwellings and swimmers sinking in upwellings, respectively, become more rare. The same trend is observed at intermediate Reynolds number [Figs. 12(d)-12(f)] as well as high Reynolds number further increases [Figs. 12(g)-12(i)], even if the spreading of values around the peak becomes increasingly narrower and limited within smaller values of $\left.\nabla_{2 \mathrm{D}}\right|_{p}$ and $w_{p}^{+}$. This may be attributed to the decorrelation between the near-surface turbulence and the bottom boundary layer, which is known to occur at high enough Reynolds number and results in lower anisotropy of the turbulence entering the surface 
region and weaker surface renewal events. In agreement with previous results in this section, the most significant modifications to the jPDF occur between the $\Psi_{L}^{+}$case (when $\Psi_{K}^{+}<1$ ) and the $\Psi_{I}^{+}$ case (when $\Psi_{K}^{+}>1$ ).

\section{Reynolds number and vertical stability number effects on directional swimming}

The surfacing dynamics examined in the previous section are in direct connection with the capability of the swimmers to orient themselves in the direction opposite to gravity. In Fig. 13 we examine the influence of $\operatorname{Re}_{\tau}$ and $\Psi$ on this capability by discussing the behavior of the vertical and streamwise component of the orientation vector, indicated as $\left\langle p_{z}\right\rangle$ and $\left\langle p_{x}\right\rangle$, respectively, as function of the distance from the free surface. Angular brackets indicate averaging over the horizontal flow directions and in time. The spanwise component of the orientation vector is not shown since it is nearly zero throughout the channel height, indicating a uniform distribution of orientations in this direction, as expected by symmetry considerations. Let us consider the low Reynolds number case first (top-row panels). The increase of vertical stability allows swimmers to reach an equilibrium orientation that leads to an increase of $\left\langle p_{z}\right\rangle$ throughout the channel with the exception of the near-wall region at the bottom: In this region, the mean shear acts to align swimmers in the horizontal direction, as indicated by the peak of $\left\langle p_{x}\right\rangle$ for the $\Psi_{H}^{+}$swimmers in Fig. 13(b).

Interestingly, this effect vanishes for the swimmers with low vertical stability, which appear to be more uniformly oriented in the flow domain. The different behavior characterizing the different sets of swimmers can be explained again in terms of small and large flow scale effects: At low gyrotaxis, the condition $\Psi_{K}^{+}<1$ is met and swimmers are destabilized "isotropically" by smallscale turbulence, which acts to disrupt directional motility; at intermediate and high gyrotaxis, the condition $\Psi_{K}^{+}>1$ is met and swimmers tend to interact preferentially with the large-scale structures of the flow, thus filtering small-scale motions. The trends just described are remarkably persistent and independent of the Reynolds number: The main $\operatorname{Re}_{\tau}$ effects that can be noted from Figs. 13(c)13(f) are a general increase of $\left\langle p_{z}\right\rangle$ and hence a decrease of $\left\langle p_{x}\right\rangle$ for all levels of gyrotaxis. We note that the peak value reached by $\left\langle p_{x}\right\rangle$, roughly equal to 0.7 , and the wall distance at which this peak occurs, roughly equal to 10 wall units, are unaffected by $\operatorname{Re}_{\tau}$.

It is worth remarking here that, in all the simulations presented, the effective rise velocity producing lower migration rates observed in Figs. 6- 8, is due to the randomisation of the swimmer orientation due to turbulence. In the context of nonactive, buoyant plankton research, there is experimental evidence that turbulence acts to increase the effective rise velocity [19]. This effect was attributed to the role of fluid inertia (added mass) [47], which is not included in the present study because it focuses on active, neutrally-buoyant organisms.

\section{CONCLUSION}

In this study, we used direct numerical simulation and Lagrangian tracking to investigate the influence of the flow Reynolds number and of the vertical stability of gyrotactic swimmers in free-surface turbulent channel flow. In this flow configuration, the flow is driven by a constant pressure gradient and is characterized by turbulent bursting phenomena at the bottom wall that then reach up to the free surface. An extensive campaign of simulations was performed for shear Reynolds number $\operatorname{Re}_{\tau}=170,510$, and 1020 to consider situations in which surface renewal mechanisms change significantly depending on the capability of near-wall bursting phenomena to bring bulk fluid to the interface. In addition, we also considered swimmers with different reorientation times, corresponding to stability number $\Psi_{L}^{+}=0.0113, \Psi_{I}^{+}=0.113$, and $\Psi_{H}^{+}=1.13$, which are representative of common motile phytoplankton species and indicate different quickness in responding to external fluctuations: Specifically, swimmers with $\Psi_{L}^{+}=0.0113$ swimmers realign in the direction opposite to gravity with a characteristic time that is longer than the Kolmogorov timescale, whereas $\Psi_{I}^{+}=0.113$ and $\Psi_{H}^{+}=1.13$ swimmers are able to reorient themselves within a fraction of the Kolmogorov timescale. 
For all gyrotactic reorientation times, swimmers are observed to move toward the free surface. However, surfacing occurs at a rate that depends on the bottom-heaviness of the swimmers: Our results indicate that vertical migration is favored when the motion of the swimmers is controlled by large-scale advection whereas small-scale turbulence hampers the ability of the swimmers to rise upwards. This opposite influence can be parameterized by means of the Kolmogorov-based stability number $\Psi_{K}^{+}=\frac{1}{2 \mathcal{B}} \tau_{K}$, with $\tau_{K}$ the Kolmogorov timescale. When the value of $\tau_{K}$ is chosen equal to the value that characterizes the dissipative flow scales at the free surface, we find that the large-scale flow structures dominate when $\Psi_{K}^{+}>1$, a condition that is met when swimmers' response to gravity is quick enough to counteract the destabilizing influence of small-scale flow structures on vertical migration; conversely, small-scale turbulence plays a role when $\Psi_{K}^{+}<1$, a condition that is met when the timescale of gyrotaxis is large compared to the local Kolmogorov timescale. At low Reynolds number, transport of swimmers by large-scale advection is favored by the fact that fluid upwellings produced by near-wall bursts maintain their spatial coherence all the way to the free surface, thus allowing efficient migration already at relatively low swimming velocities (of the order of $100 \mu \mathrm{m} / \mathrm{s}$ in the present simulations). At high Reynolds number, near-surface turbulence is less influenced by the bottom boundary layer within which it was generated and therefore higher swimming velocities (of the order of $1000 \mu \mathrm{m} / \mathrm{s}$ in the present simulations) are required to exploit large-scale advection. Once at the surface, swimmers aggregate into persistent fractal-like clusters that are controlled by the surface divergence of the flow field at small times, but then slowly evolve into highly-concentrated filaments in case of fast reorientation time (namely, $\Psi^{+}>\Psi_{L}^{+}$in the present study). Such evolution appears to be marginally influenced by the Reynolds number, at least within the range investigated here. These scaling argument based on $\Psi_{K}^{+}$may assist in the development of more accurate models for motile microorganisms in real turbulent environments, as well as more reliable simulation tools based on large-eddy simulation (LES). LES can only resolve the largest turbulent overturns, but our findings hint to the importance of developing closure models that can account for the influence of the subgrid scales on vertical migration and preferential orientation [48] if accurate predictions of surfacing and clustering are sought.

Present results apply to the case of spherical, inertialess swimmers. Previous studies have shown that even small deviations from the spherical shape $[16,32,49,50]$ and effects of nonstationary forces $[19,47]$ may induce significant differences on clustering. Elongated swimmers, in particular, are more sensitive to the local fluid shear, and tend to react more slowly to vorticity and gravity, with consequences on their tendency to preferential clustering in turbulence [16]. In turn, this may have potential consequences for the ecology of motile organisms that exploit directional swimming upon changing shape and preferential orientation. A future development of the present work is therefore to investigate the validity of the above-mentioned scaling arguments for the case of elongated swimmers, which are more strongly dominated by the local shear and less by gravity compared to spherical swimmers.

Another interesting extension of the present study is the inclusion of fluid acceleration effects on swimmer surfacing in high- $\mathrm{Re}_{\tau}$ simulations. When such effects matter, swimmer tend to sample high-vorticity regions $[17,33]$ with possible consequences on their ability to rise in upwellings and sink in downwellings.

\section{ACKNOWLEDGMENTS}

CINECA (Consorzio inter-universitario per il calcolo automatico, Italy), SNIC (Swedish National Infrastructure for Computing, Sweden), and VSC (Vienna Scientific Cluster, Austria) are gratefully acknowledged for the generous allowance of computational resources.

[1] A. Sengupta, F. Carrara, and R. Stocker, Phytoplankton can actively diversify their migration strategy in response to turbulent cues, Nature 543, 555 (2017). 
[2] E. Berdalet, F. Peters, V. Lila Koumandou, C. Roldán, O. Guadayol, and M. Estrada, Species-specific physiological response of dinoflagellates to quantified small-scale turbulence, J. Phycol. 43, 965 (2007).

[3] S. Ghorai and N. A. Hill, Gyrotactic bioconvection in three dimensions, Phys. Fluids 19, 054107 (2007).

[4] J. S. Guasto, R. Rusconi, and R. Stocker, Fluid mechanics of planktonic microorganisms, Annu. Rev. Fluid Mech. 44, 373 (2012).

[5] A. Karimi and A. M. Ardekani, Gyrotactic bioconvection at pycnoclines, J. Fluid Mech. 733, 245 (2013).

[6] N. Desai and A. M. Ardekani, Modeling of active swimmer suspensions and their interactions with the environment, Soft Matter 13, 6033 (2017).

[7] W. M. Durham, E. Climent, M. Barry, F. De Lillo, G. Boffetta, M. Cencini, and R. Stocker, Turbulence drives microscale patches of motile phytoplankton, Nat. Commun. 4, 2148 (2013).

[8] C. Torney and Z. Neufeld, Transport and Aggregation of Self-Propelled Particles in Fluid Flows, Phys. Rev. Lett. 99, 078101 (2007).

[9] W. M. Durham, J. O. Kessler, and R. Stocker, Disruption of vertical motility by shear triggers formation of thin phytoplankton layers, Science 323, 1067 (2009).

[10] W. M. Durham and R. Stocker, Thin phytoplankton layers: Characteristics, mechanisms, and consequences, Annu. Rev. Mar. Sci. 4, 177 (2012).

[11] T. J. Pedley and J. O. Kessler, Hydrodynamic phenomena in suspensions of swimming microorganisms, Annu. Rev. Fluid Mech. 24, 313 (1992).

[12] T. J. Pedley and J. O. Kessler, A new continuum model for suspensions of gyrotactic microorganisms, J. Fluid Mech. 212, 155 (1990).

[13] W. M. Durham, M. Barry, E. Climent, and R. Stocker, Gyrotaxis in a Steady Vortical Flow, Phys. Rev. Lett. 106, 238102 (2011).

[14] F. Santamaria, F. De Lillo, M. Cencini, and G. Boffetta, Gyrotactic trapping in laminar and turbulent Kolmogorov flow, Phys. Fluids 26, 111901 (2014).

[15] O. A. Croze, G. Sardina, M. Ahmed, M. Bees, and L. Brandt, Dispersion of swimming algae in laminar and turbulent channel flows: Consequences for photobioreactors, J. Royal Soc. Interface 10, 20121041 (2013).

[16] C. Zhan, G. Sardina, E. Lushi, and L. Brandt, Accumulation of motile elongated microorganisms in turbulence, J. Fluid Mech. 739, 22 (2014).

[17] F. De Lillo, M. Cencini, W. M. Durham, M. Barry, R. Stocker, E. Climent, and G. Boffetta, Turbulent Fluid Acceleration Generates Clusters of Gyrotactic Microorganisms, Phys. Rev. Lett. 112, 044502 (2014).

[18] S. Lovecchio, F. Zonta, C. Marchioli, and A. Soldati, Thermal stratification hinders gyrotactic microorganism rising in free-surface turbulence, Phys. Fluids 29, 053302 (2017).

[19] J. Ruiz, D. Macìas, and F. Peters, Turbulence increases the average settling velocity of phytoplankton cells, Proc. Natl. Acad. Sci. USA 101, 17720 (2004).

[20] M. Mashayekhpour, C. Marchioli, S. Lovecchio, E. Nemati Lay, and A. Soldati, Wind effect on gyrotactic microorganism surfacing in free-surface turbulence, Adv. Water Res. 129, 328 (2019).

[21] K. Gustavsson, F. Berglund, P. R. Jonsson, and B. Mehlig, Preferential Sampling and Small-Scale Clustering of Gyrotactic Microswimmers in Turbulence, Phys. Rev. Lett. 116, 108104 (2016).

[22] M. Borgnino, G. Boffetta, F. De Lillo, and M. Cencini, Gyrotactic swimmers in turbulence: Shape effects and role of the large-scale flow, J. Fluid Mech. 856, R1 (2018).

[23] I. Fouxon, and A. Leshansky, Phytoplankton's motion in turbulent ocean, Phys. Rev. E 92, 013017 (2015).

[24] S. Lovecchio, C. Marchioli, and A. Soldati, Time persistence of floating-particle clusters in free-surface turbulence, Phys. Rev. E 88, 033003 (2013).

[25] J. O. Kessler, Hydrodynamics focusing of motile algal cells, Nature 313, 218 (1985).

[26] R. Nagaosa and R. A. Handler, Statistical analysis of coherent vortices near a free surface in a fully developed turbulence, Phys. Fluids 15, 375 (2003).

[27] A. Kermani, H. R. Khakpour, L. Shen, and T. Igusa, Statistics of surface renewal of passive scalars in free-surface turbulence, J. Fluid Mech. 678, 379 (2011).

[28] I. Calmet and J. Magnaudet, Statistical structure of high-Reynolds-number turbulence close to the free surface of an open-channel flow, J. Fluid Mech. 474, 355 (2003). 
[29] S. Lovecchio, F. Zonta, and A. Soldati, Upscale energy transfer and flow topology in free-surface turbulence, Phys. Rev. E 91, 033010 (2015).

[30] S. Lovecchio, F. Zonta, and A. Soldati, Influence of thermal stratification on the surfacing and clustering of floaters in free-surface turbulence, Adv. Water Res. 72, 22 (2014).

[31] P. Gutiérrez and S. Aumaître, Clustering of floaters on the free surface of a turbulent flow: An experimental study, Eur. J. Mech. B-Fluids 60, 24 (2016).

[32] G. A. Voth and A. Soldati, Anisotropic particles in turbulence, Annu. Rev. Fluid Mech. 49, 249 (2017).

[33] F. De Lillo, M. Cencini, G. Boffetta, and F. Santamaria, Geotropic tracers in turbulent flows: a proxy for fluid acceleration, J. Turbul. 14, 24 (2013).

[34] M. Rashidi and S. Banerjee, Turbulent structure in free-surface channel flow, Phys. Fluids 31, 2491 (1988).

[35] Y. Pan and S. Banerjee, A numerical study of free-surface turbulence in channel flow, Phys. Fluids 7, 1649 (1995).

[36] S. Komori, H. Ueda, F. Ogino, and T. Mizushina, Turbulence structure and transport mechanism at the free surface in an open-channel flow, Int. J. Heat Mass Transfer 25, 513 (1982).

[37] S. Komori, Y. Murakami, and H. Ueda, The relationship between surface-renewal and bursting motions in an open-channel flow, J. Fluid Mech. 203, 103 (1989).

[38] R. Nagaosa and R. A. Handler, Characteristic timescales for predicting the scalar flux at a free surface in turbulent open-channel flows, AIChE J. 58, 3867 (2012).

[39] B. Eckhardt and J. Schumacher, Turbulence and passive scalar transport in a free-slip surface, Phys. Rev. E 64, 016314 (2001).

[40] S. Kumar, R. Gupta, and S. Banerjee, An experimental investigation of the characteristics of free-surface turbulence in channel flow, Phys. Fluids 10, 437 (1998).

[41] R. H. Kraichnan, Inertial ranges in two-dimensional turbulence, Phys. Fluids 10, 1417 (1967).

[42] G. K. Batchelor, Computation of the energy spectrum in homogeneous two dimensional turbulence, Phys. Fluids 12, II-233 (1969).

[43] J. R. Cressman, J. Davoudi, W. I. Goldburg, and J. Schumacher, Eulerian and Lagrangian studies in surface flow turbulence, New J. Phys. 6, 53 (2004).

[44] J. Larkin, M. M. Bandi, A. Pumir, and W. I. Goldburg, Power-law distributions of particle concentration in free-surface flows, Phys. Rev. E 80, 066301 (2009).

[45] J. Larkin, W. I. Goldburg, and M. M. Bandi, Time-evolution of a fractal distribution: Particle concentrations in free-surface turbulence, Physica D 239, 1264 (2010).

[46] P. Grassberger and I. Procaccia, Characterization of Strange Attractors, Phys. Rev. Lett. 50, 346 (1983).

[47] C. Marchioli, M. Fantoni, and A. Soldati, Influence of added mass on anomalous high rise velocity of light particles in cellular flow field. A note on the paper of Maxey (1987), Phys. Fluids 19, 098101 (2007).

[48] C. Marchioli, Large-eddy simulation of turbulent dispersed flows: A review of modeling approaches, Acta Mech. 228, 741 (2017).

[49] M. Borgnino, K. Gustavsson, F. De Lillo, G. Boffetta, M. Cencini, and B. Mehlig, Alignment of Nonspherical Active Particles in Chaotic Flows, Phys. Rev. Lett. 123, 138003 (2019).

[50] S. Lovecchio, E. Climent, R. Stocker, W. M. Durham, Chain formation can enhance the vertical migration of phytoplankton through turbulence, Sci. Adv. 5, eaaw7879 (2019). 